\title{
AN ELEMENTARY INTRODUCTION TO QUANTUM GRAPHS
}

\author{
GREGORY BERKOLAIKO
}

\begin{abstract}
We describe some basic tools in the spectral theory of Schrödinger operator on metric graphs (also known as "quantum graphs") by studying in detail some basic examples. The exposition is kept as elementary and accessible as possible. In the later sections we apply these tools to prove some results on the count of zeros of the eigenfunctions of quantum graphs.
\end{abstract}

\section{INTRODUCTION}

Studying operators of Schrödinger type on metric graphs is a growing subfield of mathematical physics which is motivated both by direct applications of the graph models to physical phenomena and by use of graphs as a simpler setting in which to study complex phenomena of quantum mechanics, such as Anderson localization, universality of spectral statistics, nodal statistics, scattering and resonances, to name but a few.

The name "quantum graphs" is most likely a shortening of the title of the article "Quantum Chaos on Graphs" by Kottos and Smilansky [36]. The model itself has been studied well before the name appeared, for example in [43, 47, 46, 51, 41].

Several reviews and monographs cover various directions within the quantum graphs research [29, 45, 40]. However, when starting a research project with students, both (post-) graduate and undergraduate, the author felt that a more elementary introduction would be helpful. The present manuscript grew out of the same preparatory lecture repeated, at different points of time, to several students. It is basically a collection of minimal examples of quantum graphs which already exhibit behavior typical to larger graphs. We supply the examples with pointers to the more general facts and theorems. Only in the last sections we explore a research topic (the nodal statistics on graphs) in some depth.

For obvious reasons the pointers often lead to the monograph [15]; the notation is kept in line with that book, too.

\section{SChröDinger EQUATION ON A METRIC GRAPH}

Consider a graph $\Gamma=(V, E)$, where $V$ is the set of vertices and $E$ is the set of edges. Each edge connects a pair of vertices; we allow more than one edge running between any two vertices. We also allow edges connecting vertices to themselves (loops). This freedom creates some notational difficulties, so we ask the reader to be flexible and forgiving.

Each edge $e$ is assigned a positive length $L_{e}$ and is thus identified with an interval $\left[0, L_{e}\right]$ (the direction is chosen arbitrarily and is irrelevant to the resulting theory). This makes $\Gamma$ a metric graph. Now a function on a graph is just a collection of functions defined on individual edges. 
The eigenvalue equation for the Schrödinger operator is

$$
-\frac{d^{2} f}{d x^{2}}+V(x) f(x)=\lambda f(x)
$$

which is to be satisfied on every edge, in addition to the vertex matching conditions as follows

$$
\begin{aligned}
& f(x) \text { is continuous, } \\
& \sum_{e \sim v} \frac{d f}{d x}(v)=0 .
\end{aligned}
$$

The continuity means that the values at the vertex agree among all functions living on the edges attached (or incident) to the vertex. In the second condition (often called current conservation condition), the sum is over all edges attached to the vertex and the derivative are all taken in the same direction: from the vertex into the edge. A looping edge contributes two terms to the sum, one for each end of the edge.

The function $V(x)$ is called the electric potential but we will set it identically to zero in all of the examples below. Vertex conditions (2)-(3) are called Neumann conditions 1 ; they can be generalized significantly, but before we give any more theory, let us consider some examples.

2.1. Example: a trivial graph - an interval. An interval $[0, L]$ is the simplest example of a graph; it has two vertices (the endpoints of the interval) and one edge. The continuity condition is empty at every vertex since there is only one edge. The current conservation condition at the vertex 0 becomes

$$
f^{\prime}(0)=0
$$

and at the vertex $L$ becomes

$$
-f^{\prime}(L)=0 .
$$

The minus sign appeared because we agreed to direct the derivatives into the edge; of course it is redundant in this particular case.

Let $V(x) \equiv 0$ and consider first the positive eigenvalues, $\lambda>0$. The eigenvalue equation becomes

$$
-f^{\prime \prime}=k^{2} f,
$$

where for convenience we substituted $\lambda=k^{2}$. This is a second order linear equation with constant coefficients which for $k>0$ is readily solved by

$$
f(x)=C_{1} \cos (k x)+C_{2} \sin (k x) .
$$

Applying the first vertex condition $f^{\prime}(0)=0$ we get $C_{2}=0$ and $f(x)=C_{1} \cos (k x)$. The second vertex condition becomes

$$
C_{1} k \sin (k L)=0,
$$

which imposes a condition on $k$ but does nothing to determine $C_{1}$ (naturally we are not interested in the trivial solution $f(x) \equiv 0)$. We thus get the eigenvalues $\lambda=k^{2}=(\pi n / L)^{2}$, $n=1,2, \ldots$ with the corresponding eigenfunctions $f(x)=\cos (\pi n x / L)$ defined up to an overall constant multiplier (as befits eigenvectors and eigenfunctions).

\footnotetext{
${ }^{1}$ Other names present in the literature are "Kirchhoff", "Neumann-Kirchhoff", "standard", "natural" etc.
} 
There is one other eigenvalue in the spectrum that we missed: $\lambda=0$ with the eigenfunction $f(x) \equiv 1$. While this agrees with the above formulas with $n=0$, the premise of equation (7) is no longer correct when $\lambda=0$.

Exercise 2.1. Solve the eigenvalue equation with $\lambda<0$ and show that the vertex conditions (4) and (5) are never satisfied simultaneously (ignore the trivial solution $f(x) \equiv 0)$.

Exercise 2.2. Integrate by parts the scalar product

$$
\left\langle f,-f^{\prime \prime}\right\rangle=\int_{0}^{L} \overline{f(x)}\left(-f^{\prime \prime}(x)\right) d x,
$$

to obtain an expression that is obviously non-negative, and thus show that it is not necessary to solve (6) to conclude that there are no negative eigenvalues.

We did not try to look for complex eigenvalues. This is because the Schrödinger operator we defined is self-adjoint (see Thm 1.4.4 of [15]) and therefore has real spectrum. The spectrum in the above example is discrete: all eigenvalues are isolated and of finite multiplicity. This is true for any graph which is compact (has finitely many edges, all of which have finite length), see Thm 3.1.1 of [15]. The proof outlined in Exercise 2.2 works for general graphs with Neumann conditions at all vertices. The multiplicity of the eigenvalue 0 in the spectrum can be shown to equal the number of the connected components of the graph.

2.2. Example: a trivializable graph with a vertex of degree two. Consider now a graph consisting of two consecutive intervals, $\left[0, L_{1}\right]$ and $\left[L_{1}, L_{1}+L_{2}\right]$. We do not really have to parametrize the edges starting from 0, so in this example we will employ the "natural" parametrization.

Denote the components of eigenfunction living on the two intervals by $f_{1}$ and $f_{2}$ correspondingly. Solving the equation on the first edge and enforcing the Neumann condition at 0 results in $f_{1}(x)=C \cos (k x)$. The conditions at the point $L_{1}$ are

$$
\begin{aligned}
& f_{1}\left(L_{1}\right)=f_{2}\left(L_{1}\right), \\
& -f_{1}^{\prime}\left(L_{1}\right)+f_{2}^{\prime}\left(L_{1}\right)=0 .
\end{aligned}
$$

Now, by uniqueness theorem for second order differential equations, the solution on the second edge is fully determined by its value at $L_{1}$ and the value of its derivative. Thus the solution is still $f_{2}(x)=C \cos (k x)$ and there is no change in the solution happening at $L_{1}$. We could have considered the interval $\left[0, L_{1}+L_{2}\right]$ without introducing the additional vertex at $L_{1}$. This obviously generalizes to the following rule: having a Neumann vertex of degree 2 is equivalent to having an uninterrupted edge.

This rule is useful, for example, for when one wants to program a looping edge but is troubled by the notational difficulties of loops or multiple edges. In this case a looping edge can be implemented as a triangle with two "dummy" vertices of degree two.

2.3. Example: star graph with Neumann endpoints. Consider now a first non-trivial example: a star graph with 3 edges meeting at a central vertex, see Fig. 1. Parametrizing 


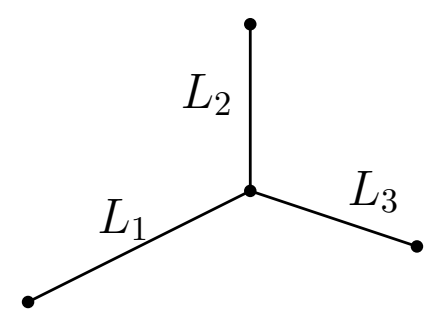

FiguRE 1. A star graph with three edges.

the edges from the endpoints towards the central vertex, we get

$$
\begin{aligned}
& -f_{1}^{\prime \prime}=k^{2} f_{1}, \quad-f_{2}^{\prime \prime}=k^{2} f_{2}, \quad-f_{3}^{\prime \prime}=k^{2} f_{3}, \\
& f_{1}^{\prime}(0)=0, \quad f_{2}^{\prime}(0)=0, \quad f_{3}^{\prime}(0)=0, \\
& f_{1}\left(L_{1}\right)=f_{2}\left(L_{2}\right)=f_{3}\left(L_{3}\right), \\
& -f_{1}^{\prime}\left(L_{1}\right)-f_{2}^{\prime}\left(L_{2}\right)-f_{3}^{\prime}\left(L_{3}\right)=0,
\end{aligned}
$$

where in addition to the already familiar equations $(12)$ and $(13)$ (in three copies), we have continuity condition at the central vertex in equation (14) and current conservation at the central vertex in equation $(15)$. Note that in equation $(12)$, the eigenvalue $k^{2}$ is the same on all three edges.

Equations 12 - 13 are solved by

$$
f_{1}(x)=A_{1} \cos (k x), \quad f_{2}(x)=A_{2} \cos (k x), \quad f_{3}(x)=A_{3} \cos (k x),
$$

for some constants $A_{1}, A_{2}$ and $A_{3}$. Now the remaining two equations become, after a minor simplification,

$$
\begin{aligned}
& A_{1} \cos \left(k L_{1}\right)=A_{2} \cos \left(k L_{2}\right)=A_{3} \cos \left(k L_{3}\right), \\
& A_{1} \sin \left(k L_{1}\right)+A_{2} \sin \left(k L_{2}\right)+A_{3} \sin \left(k L_{3}\right)=0 .
\end{aligned}
$$

Dividing equation (18) by (17) cancels the unknown constants, resulting in

$$
\tan \left(k L_{1}\right)+\tan \left(k L_{2}\right)+\tan \left(k L_{3}\right)=0 .
$$

Squares of the roots $k$ of this equation (which cannot be solved explicitly except when all $L \mathrm{~s}$ are equal) are the eigenvalues of the star graph.

Exercise 2.3. We ignored the possibility that one or more of the cosines in equation (17) are zero. Show that the more robust (but much longer!) version of equation (19) is

$$
\begin{aligned}
\sin \left(k L_{1}\right) \cos \left(k L_{2}\right) \cos \left(k L_{3}\right)+\cos \left(k L_{1}\right) \sin \left(k L_{2}\right) \cos \left(k L_{3}\right) & \\
& +\cos \left(k L_{1}\right) \cos \left(k L_{2}\right) \sin \left(k L_{3}\right)=0 .
\end{aligned}
$$

Moreover, the order of the root $k$ of 20 is equal to the dimension of the corresponding eigenspace.

For example, if $L_{1}=L_{2}=L_{3}=\pi / 2$, the left-hand side of $(20)$ vanishes at $k=1$ to the second order. This corresponds to two linearly independent solutions,

$$
\left(f_{1}, f_{2}, f_{3}\right)=(\cos (x),-\cos (x), 0) \text { and }(\cos (x), 0,-\cos (x)) .
$$

There is actually a lot more that can be (and will be said) about this simple graph, but we first need to extend the set of possible vertex conditions that we consider. 

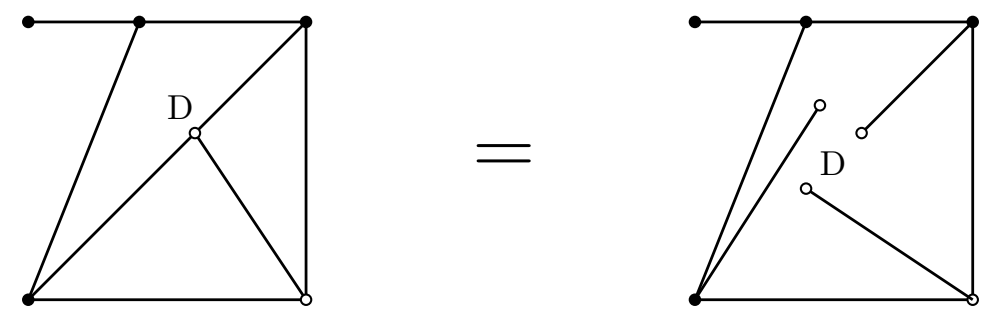

Figure 2. A Dirichlet condition imposed at a vertex of degree $d=3$ (here and in subsequent figures, the Dirichlet vertices are denoted by empty circles) is equivalent to splitting the vertex into three and imposing the condition at every new vertex.

\section{DiRICHLET CONDITION}

Another possible vertex condition which is compatible with self-adjointness of the Schrödinger operator is the so-called Dirichlet condition,

$$
f_{e}(v)=0 \text { for all } e \text { incident to } v \text {. }
$$

It is usually used only at vertices of degree 1 for the following reason. A Dirichlet condition imposed at a vertex of degree 2 or more fails to relate in any way the individual functions living on the incident edges. Thus a graph with a Dirichlet condition at a vertex $v$ of degree $d_{v}$ is equivalent to a graph with $v$ substituted with $d_{v}$ vertices of degree one, see Fig. 2.

Note that the difference between a Neumann and a Dirichlet condition at a vertex of degree $d_{v}$ is minimal: the current conservation condition is substituted with the condition that one of the function values is equal to zero; the rest is taken care of by continuity.

Exercise 3.1. Show that the eigenvalues of the interval $[0, L]$ with Dirichlet conditions at both ends are given by $\lambda_{n}=(\pi n / L)^{2}, n=1,2, \ldots$ with the eigenfunctions $f^{(n)}(x)=\sin (\pi n x / L)$.

3.1. Example: a star graph with Dirichlet conditions at endpoints. Consider a star graph with $N$ edges. We parametrize the edges from the endpoints towards the central vertex, as before. Solving the eigenvalue equation $-f^{\prime \prime}=k^{2} f$ and imposing the Dirichlet condition at $x=0$ results in $f_{i}(x)=A_{i} \sin (k x)$, where the constant $A_{i}$ depends on the edge.

At the central vertex we have

$$
\begin{aligned}
& A_{1} \sin \left(k L_{1}\right)=\ldots=A_{N} \sin \left(k L_{N}\right), \\
& A_{1} \cos \left(k L_{1}\right)+\ldots+A_{N} \cos \left(k L_{N}\right)=0 .
\end{aligned}
$$

If we assume that the lengths $L_{i}$ are incommensurate, we will not be missing any roots by dividing equation (24) by (23), leading to the eigenvalue condition

$$
\sum_{i=1}^{N} \cot \left(k L_{i}\right)=0 .
$$

This condition is very similar to equation (19) we derived for the star graph with Neumann conditions at the endpoints. However, it is now easier to see a connection between the star graph and the eigenvalue problem of an interval.

The left-hand side of equation (25) has derivative of constant sign (negative) except at the poles $k \in\left\{n \pi / L_{i}\right\}, i=1, \ldots, N, n \in \mathbb{Z}$. Therefore, between each pair of consecutive 


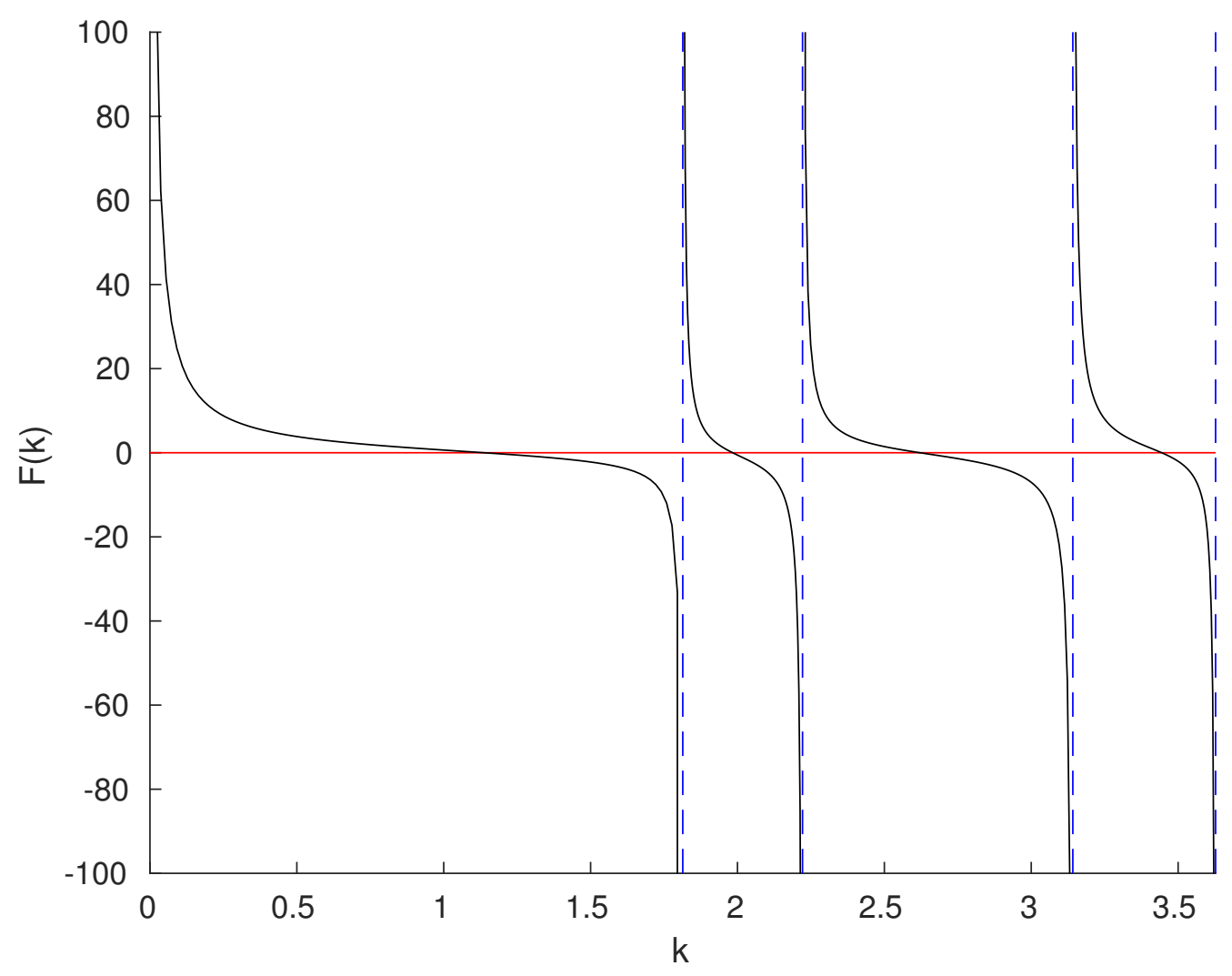

Figure 3. Plot of the right-hand side of 25 with 3 edges of lengths $1, \sqrt{2}$ and $\sqrt{3}$. It is a function that decreases monotonely between each pair of its poles. The poles visible on the plot are $0, \pi / \sqrt{3}, \pi / \sqrt{2}, \pi$ and $2 \pi / \sqrt{3}$.

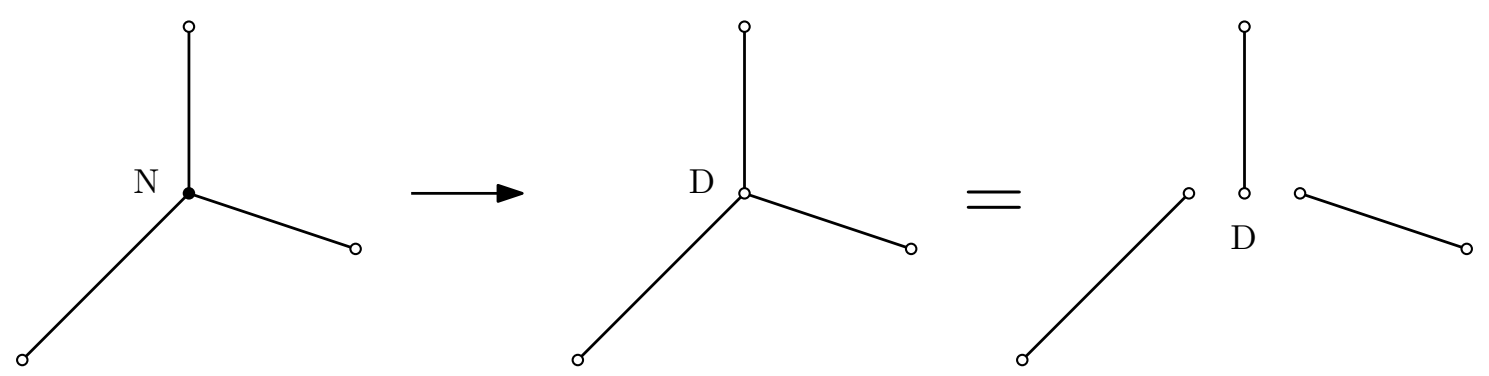

Figure 4. A star graph with the central vertex condition changed to Dirichlet splits into a collection of intervals.

poles there is a single root of equation (25), see Fig. 3. The poles can be interpreted as the square roots of the eigenvalues of the individual edges of the graph, see Exercise 3.1 . Furthermore, the collection of the edges with Dirichlet conditions can be obtained from the original star graph by changing the central vertex condition from Neumann to Dirichlet, see Fig. 4. As we mentioned already, this can be effected by changing only one equation in the Neumann conditions, which is a rank-one perturbation. To summarize, we found that there is exactly one eigenvalue of a star graph between any two consecutive eigenvalues of its rank 1 perturbation. 

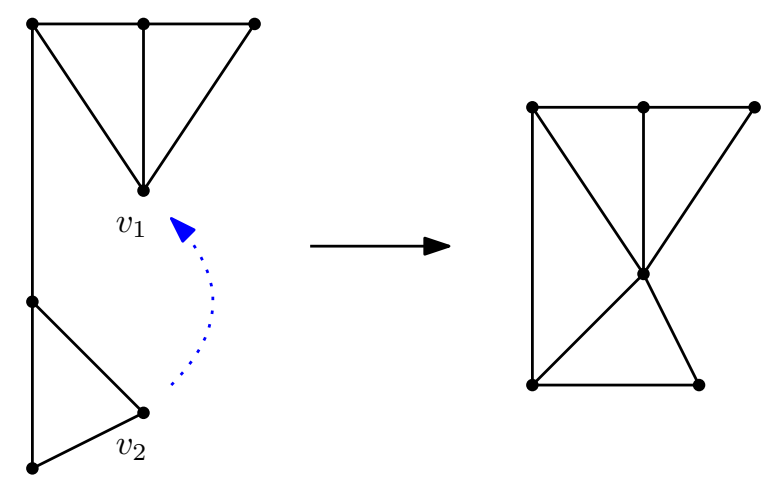

FiguRE 5. The operation of merging two vertices into one.

\section{INTERLACING INEQUALITIES}

Naturally, the observation of Section 3.1 applies not only to star graphs but to any graphs with discrete spectrum.

Lemma 4.1 (Neumann-Dirichlet interlacing). Let $\Gamma_{0}$ be a quantum graph with a vertex $v$ which is endowed with Neumann conditions. Let $\Gamma_{\infty}$ denote the graph obtained by changing the conditions at $v$ to Dirichlet. Numbering the eigenvalues of both graphs in ascending order starting from 1, we have

$$
\lambda_{n}\left(\Gamma_{0}\right) \leq \lambda_{n}\left(\Gamma_{\infty}\right) \leq \lambda_{n+1}\left(\Gamma_{0}\right) \leq \lambda_{n+1}\left(\Gamma_{\infty}\right)
$$

An equality between a Neumann and a Dirichlet eigenvalue is possible only if the eigenspace of the Neumann eigenvalue contains a function vanishing at $v$, or, equivalently, the eigenspace of the Dirichlet eigenvalue contains a function satisfying the current conservation condition.

The proof, which can be found in [14, 15], uses the standard arguments built upon the minimax characterisation of eigenvalues of a self-adjoint operator. It is analogous to the proofs of Cauchy's Interlacing Theorem or rank-one perturbations for matrices, see, for example [32].

The following exercise contains an application of Lemma 4.1 to the nodal count of eigenfunctions.

Exercise 4.2. Show that if the $n$-th eigenvalue of a star graph with Dirichlet endpoints is simple and the corresponding eigenfunction is non-vanishing at the central vertex, the eigenfunction has precisely $n-1$ zeros in the interior of the graph.

This statement can be obtained by combining the strict version of inequality (26) with the following observation. If $\lambda$ satisfies $\lambda_{n}(I) \leq \lambda \leq \lambda_{n+1}(I)$, where $I$ is the interval [0,L] with Dirichlet boundary conditions, then $f(x)=\sin (\sqrt{\lambda} x)$ has $n$ zeros on the interval $(0, L)$.

A more general version of this statement holds for tree graphs. This theorem has a rich history, originally appearing in [44] and [49]; the shortest proof along the lines outlined in Exercise 4.2 appeared in [14] and in Section 5.2.2 of [15]. 
Another useful interlacing inequality arises when we join two Neumann vertices to form a single Neumann vertex, see Fig. 5. Since the change in the vertex conditions can be described as imposing another continuity equality|2, we expect the eigenvalues to increase as a result.

Lemma 4.3. Let $\Gamma$ be a quantum graph (not necessarily connected) with two vertices $v_{1}$ and $v_{2}$ with Neumann conditions. Modify the graph by merging the two vertices into one, to obtain the graph $\Gamma^{\prime}$. Then

$$
\lambda_{n}(\Gamma) \leq \lambda_{n}\left(\Gamma^{\prime}\right) \leq \lambda_{n+1}(\Gamma) .
$$

An equality between an eigenvalue of $\Gamma$ and an eigenvalue of $\Gamma^{\prime}$ is only possible if the eigenspace of $\Gamma$ contains an eigenfunction whose values at $v_{1}$ and $v_{2}$ are equal or, equivalently, the eigenspace of $\Gamma^{\prime}$ contains an eigenfunction which additionally satisfy the current conservation condition with respect to the subset $E_{v_{1}}$ of the edges incident to vertex $v_{1}$ in the graph $\Gamma$.

This lemma and Lemma 4.1 have many applications to counting zeros of a graph's eigenfunctions, one of which will be presented in Section 7.3. Another application is to the eigenvalue counting which is the subject of the next section.

4.1. An application to eigenvalue counting: Weyl's law. Let us define the eigenvalue counting function $N_{\Gamma}(k)$ as the number of eigenvalues of the graph $\Gamma$ which are smaller than $k^{2}$,

$$
N_{\Gamma}(k)=\#\left\{\lambda \in \sigma(\Gamma): \lambda \leq k^{2}\right\} .
$$

This number is guaranteed to be finite since the spectrum of a quantum graph is discrete and bounded from below (Sec 3.1.1 and Thm 1.4.19 of [15]). We count the eigenvalues in terms of $k=\sqrt{\lambda}$ as this is more convenient and can be easily related back to $\lambda$.

The counting function $N_{\Gamma}(k)$ grows linearly in $k$, with the slope proportional to the "size" of the graph. This type of result is known as the "Weyl's Law".

Lemma 4.4. Let $\Gamma$ be a graph with Neumann or Dirichlet conditions at every vertex. Then

$$
N(k)=\frac{\mathcal{L}}{\pi} k+O(1)
$$

where $\mathcal{L}=L_{1}+\ldots+L_{|E|}$ is the total length of the graph's edges and the remainder term is bounded above and below by constants independent of $k$.

Proof. Let us first consider an interval of length $L$ with Dirichlet conditions. We know the eigenvalues are $(\pi n / L)^{2}, n \in \mathbb{N}$, therefore we can express $N_{L}(k)$ using the integer part function,

$$
N_{L}(k)=\left\lfloor\frac{k L}{\pi}\right\rfloor
$$

and thus bound it,

$$
\frac{L}{\pi} k-1 \leq N_{L}(k) \leq \frac{L}{\pi} k
$$

\footnotetext{
${ }^{2}$ This is not entirely correct, as the two current conservations conditions are also relaxed into one. However, in terms of quadratic forms, which impose the current conservation automatically, the change is indeed a one-dimensional reduction of the domain of the form.
} 


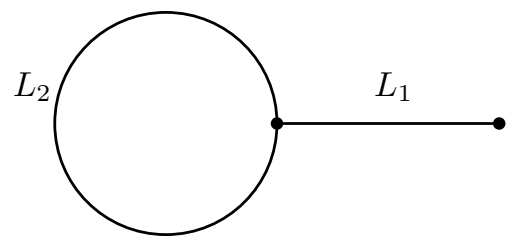

Figure 6. A lasso (or lollipop) graph, consisting of an edge and a loop.

Let us now consider the setting of Lemma 4.1: $\Gamma_{0}$ is a quantum graph with a vertex $v$ which is endowed with Neumann conditions and $\Gamma_{\infty}$ is the graph obtained by changing the conditions at $v$ to Dirichlet. Inequality 26 can be rewritten as

$$
N_{\Gamma_{\infty}}(k) \leq N_{\Gamma_{0}}(k) \leq N_{\Gamma_{\infty}}(k)+1 .
$$

Starting with a graph $\Gamma$, we can change conditions at every vertex to Dirichlet. Applying the interlacing inequality $|V|$ times (or less, if some vertices are already Dirichlet), we get

$$
N_{\Gamma_{D}}(k) \leq N_{\Gamma}(k) \leq N_{\Gamma_{D}}(k)+|V|,
$$

where by $\Gamma_{D}$ we denote the graph with every vertex conditions changed to Dirichlet. The graph $\Gamma_{D}$ is just a collection of disjoint intervals, each with Dirichlet conditions at the endpoints. The eigenvalue spectrum of $\Gamma_{D}$ is the union (in the sense of multisets) of the spectra of the intervals; the counting function is the sum of the interval counting functions. By adding $|E|$ inequalities of type $(30)$, we get

$$
\frac{L_{1}+\ldots+L_{|E|}}{\pi} k-|E| \leq N_{\Gamma_{D}}(k) \leq \frac{L_{1}+\ldots+L_{|E|}}{\pi} k,
$$

leading to the final estimate

$$
\frac{\mathcal{L}}{\pi} k-|E| \leq N_{\Gamma}(k) \leq \frac{\mathcal{L}}{\pi} k+|V|
$$

Remark 4.5. The bounds on the remainder term in the Weyl's law for a graph obtained in the proof are of order $|E|$. However, numerically it appears that the counting function follows the Weyl's term much more closely. Getting the optimal bound remains an open question at the time of writing.

\section{SeCular Determinant}

We will now describe another procedure for deriving an equation for the eigenvalues of a quantum graph. Before we describe the general case, we shall tackle a simple but useful example.

5.1. Example: lasso (lollipop) graph. Consider the graph depicted in Fig. 6, an edge attached to a loop. We will impose Neumann conditions at both the attachment point and the endpoint of the edge.

Let the edge be parametrized by $\left[0, L_{1}\right]$ with 0 corresponding to the attachment point and the loop be parametrized by $\left[0, L_{2}\right]$. The solution of the eigenvalue equation $-f^{\prime \prime}=k^{2} f$ on the edge can be written as

$$
f_{1}(x)=a_{1} e^{i k x}+a_{\overline{1}} e^{i k\left(L_{1}-x\right)}
$$



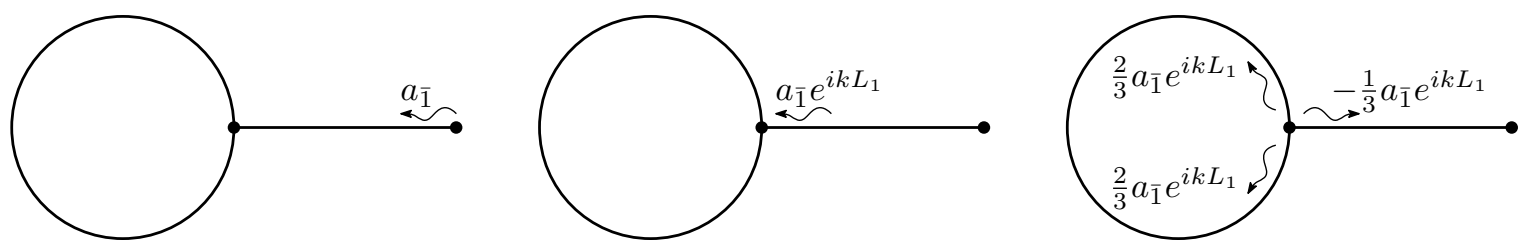

FiguRE 7. Scattering of a wave on a vertex. The waves leaves the endpoint with amplitude $a_{\overline{1}}$, acquires the phase $e^{i k L_{1}}$ by traversing the edge and scatters in three directions on the vertex.

valid as long as $k \neq 0$ (we take care of this special case separately). The Neumann condition at the endpoint leads to

$$
f_{1}^{\prime}\left(L_{1}\right)=i k a_{1} e^{i k L_{1}}-i k a_{\overline{1}}=0
$$

and therefore

$$
a_{\overline{1}}=a_{1} e^{i k L_{1}} .
$$

The solution on the loop we express similarly as

$$
f_{2}(x)=a_{2} e^{i k x}+a_{\overline{2}} e^{i k\left(L_{2}-x\right)},
$$

At the attachment point, the continuity condition reads

$$
a_{1}+a_{\overline{1}} e^{i k L_{1}}=a_{2}+a_{\overline{2}} e^{i k L_{2}}=a_{\overline{2}}+a_{2} e^{i k L_{2}},
$$

while the current conservation is

$$
f_{1}^{\prime}(0)+f_{2}^{\prime}(0)-f_{2}^{\prime}\left(L_{2}\right)=0,
$$

which, after simplification, yields

$$
a_{1}-a_{\overline{1}} e^{i k L_{1}}+a_{2}-a_{\overline{2}} e^{i k L_{2}}+a_{\overline{2}}-a_{2} e^{i k L_{2}}=0 .
$$

Rearranging equations $(39)$ and $(41)$ we get the system

$$
\begin{aligned}
& a_{1}=-\frac{1}{3} a_{\overline{1}} e^{i k L_{1}}+\frac{2}{3} a_{\overline{2}} e^{i k L_{2}}+\frac{2}{3} a_{2} e^{i k L_{2}}, \\
& a_{2}=\frac{2}{3} a_{\overline{1}} e^{i k L_{1}}+\frac{2}{3} a_{2} e^{i k L_{2}}-\frac{1}{3} a_{\overline{2}} e^{i k L_{2}}, \\
& a_{\overline{2}}=\frac{2}{3} a_{\overline{1}} e^{i k L_{1}}-\frac{1}{3} a_{2} e^{i k L_{2}}+\frac{2}{3} a_{\overline{2}} e^{i k L_{2}} .
\end{aligned}
$$

This system has an interesting "dynamical" interpretation, see Fig. 7. Take, for example, the coefficient $a_{\overline{1}}$ and interpret it as the amplitude of the plain wave leaving the endpoint vertex in the direction of the loop. Traversing the edge (of length $L_{1}$ ), it acquires the phase factor of $e^{i k L_{1}}$. Hitting the vertex, it scatters in three directions: back into the edge with back-scattering amplitude $-1 / 3$ contributing to the right-hand side of equation (42), and forward into the two ends of the loop, with forward scattering amplitude $2 / 3$, contributing to equations (43) and 44.

The amplitudes $a_{1}, a_{2}$ and $a_{\overline{2}}$ can be interpreted similarly and undergo the same process. Equations (37) and (42)-(44) can be interpreted as describing a stationary state of such a 
dynamical process. They can be written as

$$
\left(\begin{array}{cccc}
0 & -\frac{1}{3} & \frac{2}{3} & \frac{2}{3} \\
1 & 0 & 0 & 0 \\
0 & \frac{2}{3} & \frac{2}{3} & -\frac{1}{3} \\
0 & \frac{2}{3} & -\frac{1}{3} & \frac{2}{3}
\end{array}\right)\left(\begin{array}{cccc}
e^{i k L_{1}} & 0 & 0 & 0 \\
0 & e^{i k L_{1}} & 0 & 0 \\
0 & 0 & e^{i k L_{2}} & 0 \\
0 & 0 & 0 & e^{i k L_{2}}
\end{array}\right)\left(\begin{array}{l}
a_{1} \\
a_{\overline{1}} \\
a_{2} \\
a_{\overline{2}}
\end{array}\right)=\left(\begin{array}{c}
a_{1} \\
a_{\overline{1}} \\
a_{2} \\
a_{\overline{2}}
\end{array}\right)
$$

Defining two matrices

$$
S=\left(\begin{array}{cccc}
0 & -\frac{1}{3} & \frac{2}{3} & \frac{2}{3} \\
1 & 0 & 0 & 0 \\
0 & \frac{2}{3} & \frac{2}{3} & -\frac{1}{3} \\
0 & \frac{2}{3} & -\frac{1}{3} & \frac{2}{3}
\end{array}\right) \quad \text { and } \quad D=\left(\begin{array}{cccc}
e^{i k L_{1}} & 0 & 0 & 0 \\
0 & e^{i k L_{1}} & 0 & 0 \\
0 & 0 & e^{i k L_{2}} & 0 \\
0 & 0 & 0 & e^{i k L_{2}}
\end{array}\right)
$$

we can interpret (45) as saying that the matrix $S D(k)$ has 1 as its eigenvalue. Moreover, each eigenvector of the eigenvalue 1 gives rise to a solution of the eigenvalue equation for the original differential operator, via equations (35) and (38). In other words, the geometric multiplicity of the eigenvalue 1 of the matrix $S D(k)$ is equal to the geometric multiplicity of the eigenvalue $k^{2}$ of the differential operator $-\frac{d^{2}}{d x^{2}}$. Furthermore, both matrices $S$ and $D(k)$ are unitary, thus for their product $S D(k)$, the algebraic and geometric multiplicity coincide. The only point where this relationship can break down is at $k=0$; this is because solutions (35) and (38) are not valid at this point.

To summarize, we have the following criterion: the value $k^{2} \neq 0$ is an eigenvalue of the lasso quantum graph if and only if $k$ is a root of the equation

$$
\Sigma(k):=\operatorname{det}(I-S D(k))=0 .
$$

The multiplicity of $k^{2}$ in the spectrum of the graph coincides with the multiplicity of $k$ as a root of $\Sigma(k)$. The function $\Sigma(k)$ is called the secular determinant of the graph.

To finish this example, we mention that explicit evaluation results in

$$
\Sigma(k)=\frac{1}{3}\left(z_{2}-1\right)\left(3 z_{1}^{2} z_{2}-z_{1}^{2}+z_{2}-3\right), \quad \text { where } z_{1}=e^{i k L_{1}}, z_{2}=e^{i k L_{2}} .
$$

We will understand the reason for the factorization of $\Sigma(k)$ in Section 6. Note that the value $k=0$ is a double root of $\Sigma(k)$ whereas $\lambda=0$ is a simple eigenvalue of the graph (with constant as the eigenfunction).

5.2. Secular determinant for a general Neumann graph. Let us now consider a general vertex with Neumann conditions and $d$ edges incident to it. Writing the solution on $j$-th edge as

$$
f_{j}(x)=a_{j} e^{i k x}+a_{j} e^{i k\left(L_{j}-x\right)}
$$

we get from the vertex conditions

$$
\begin{array}{r}
a_{1}+a_{\overline{1}} e^{i k L_{1}}=\ldots=a_{d}+a_{\bar{d}} e^{i k L_{d}}, \\
\sum_{j=1}^{d} a_{j}-\sum_{j=1}^{d} a_{\bar{j}} e^{i k L_{j}}=0 .
\end{array}
$$


For any $n, 1 \leq n \leq d$, equations (50) imply

$$
\sum_{j=1}^{d} a_{j}+\sum_{j=1}^{d} a_{\bar{j}} e^{i k L_{j}}=d\left(a_{n}+a_{\bar{n}} e^{i k L_{n}}\right) .
$$

Subtracting from this equation (51) and solving for $a_{n}$ results in

$$
a_{n}=-a_{\bar{n}} e^{i k L_{n}}+\frac{2}{d} \sum_{j=1}^{d} a_{\bar{j}} e^{i k L_{j}}
$$

which is a generalization of both (37) and (42)-(44), with $d=1$ and $d=3$ correspondingly.

Now, it is clear how to generalize the matrices $S$ and $D(k)$ in equation 46 . Every edge of the graph gives rise to two directed edges which inherit the length of the edge. The two directed edges corresponding to the same undirected edge are reversals of each other. The reversal of a directed edge $j$ is denoted by $\bar{j}$. Consider the $2|E|$-dimensional complex space, with dimensions indexed by the directed edges. The matrix $D(k)$ is diagonal with entries

$$
D(k)_{j, j}=e^{i k L_{j}}
$$

while the matrix $S$ has the entries

$$
S_{j^{\prime}, j}= \begin{cases}\frac{2}{d_{v}}-1, & \text { if } j^{\prime}=\bar{j}, \\ \frac{2}{d_{v}}, & \text { if } j^{\prime} \text { follows } j \text { and } j^{\prime} \neq \bar{j}, \\ 0, & \text { otherwise. }\end{cases}
$$

The edge $j^{\prime}$ follows $j$ if the end-vertex of $j$ is the start vertex of $j^{\prime} ; d_{v}$ denotes the degree of the end-vertex of $j$. The matrix $S$ is sometimes called the bond scattering matrix.

Exercise 5.1. Prove that the matrix $S$ defined by $(55)$ on a graph is unitary.

As before, every eigenvector of $S D(k)$ with $k \neq 0$ corresponds to an eigenfunction of the graph via equation (49). We therefore have the following theorem.

Theorem 5.2. Consider a graph with Neumann conditions at every vertex. The value $\lambda=$ $k^{2} \neq 0$ is an eigenvalue of the operator $-d^{2} / d x^{2}$ if and only if $k$ is the solution of

$$
\Sigma(k):=\operatorname{det}(I-S D(k))=0,
$$

where $S$ and $D(k)$ are defined in equations (54) and (55).

Exercise 5.3. Incorporate vertices of degree 1 with Dirichlet conditions by showing that backscattering from such a vertex has amplitude -1 (in contrast with 1 from a Neumann vertex, see the first case of (55) with $d=1$ ). This matches with the classic reflection principle of the wave equation on an interval.

Remark 5.4. Another method used to prove the Weyl's Law (Lemma 4.4) is to observe [30] that the eigenvalues of the matrix $S D(k)$ lie on the unit circle and move in the counterclockwise direction as $k$ is increased. There are $2|E|$ of the eigenvalues and their average angular speed can be calculated to be $\mathcal{L} /|E|$. Thus the frequency of an eigenvalue crossing the positive real axis is the average speed times the number of eigenvalues divided by the length of the circle, giving $2|E| \times \mathcal{L} /|E| /(2 \pi)=\mathcal{L} / \pi$. 
5.3. Example: secular determinant of star graphs with three edges. Consider again a star graph with 3 edges and Neumann conditions everywhere. Ordering the directed edges as $[1,2,3, \overline{1}, \overline{2}, \overline{3}]$, where $j$ is directed away from the central vertex and $\bar{j}$ is directed towards the central vertex, the bond scattering matrix is

$$
S=\left(\begin{array}{cccccc}
0 & 0 & 0 & -1 / 3 & 2 / 3 & 2 / 3 \\
0 & 0 & 0 & 2 / 3 & -1 / 3 & 2 / 3 \\
0 & 0 & 0 & 2 / 3 & 2 / 3 & -1 / 3 \\
1 & 0 & 0 & 0 & 0 & 0 \\
0 & 1 & 0 & 0 & 0 & 0 \\
0 & 0 & 1 & 0 & 0 & 0
\end{array}\right)
$$

Evaluating the secular determinant $\Sigma(k)$ from equation (56), we get

(58) $\Sigma(k)=-z_{1}^{2} z_{2}^{2} z_{3}^{2}-\frac{1}{3}\left(z_{1}^{2} z_{2}^{2}+z_{2}^{2} z_{3}^{2}+z_{3}^{2} z_{1}^{2}\right)+\frac{1}{3}\left(z_{1}^{2}+z_{2}^{2}+z_{3}^{2}\right)+1, \quad$ where $z_{j}=e^{i k L_{j}}$.

Dividing this expression by $i z_{1} z_{2} z_{3}=i e^{i k\left(L_{1}+L_{2}+L_{3}\right)}$ and using the Euler's formula we can transform equation (58) to the form (20) obtained previously (up to a constant).

According to Exercise 5.3, supplying the star graph with Dirichlet conditions at the endpoints results in the bond scattering matrix

$$
S=\left(\begin{array}{cccccc}
0 & 0 & 0 & -1 / 3 & 2 / 3 & 2 / 3 \\
0 & 0 & 0 & 2 / 3 & -1 / 3 & 2 / 3 \\
0 & 0 & 0 & 2 / 3 & 2 / 3 & -1 / 3 \\
-1 & 0 & 0 & 0 & 0 & 0 \\
0 & -1 & 0 & 0 & 0 & 0 \\
0 & 0 & -1 & 0 & 0 & 0
\end{array}\right)
$$

and the secular determinant

$$
\Sigma(k)=z_{1}^{2} z_{2}^{2} z_{3}^{2}-\frac{1}{3}\left(z_{1}^{2} z_{2}^{2}+z_{2}^{2} z_{3}^{2}+z_{3}^{2} z_{1}^{2}\right)-\frac{1}{3}\left(z_{1}^{2}+z_{2}^{2}+z_{3}^{2}\right)+1 .
$$

Again, dividing it by $z_{1} z_{2} z_{3}=e^{i k\left(L_{1}+L_{2}+L_{3}\right)}$ brings it close to the previously obtained form, equation (25), namely to

$$
\begin{aligned}
e^{-i k\left(L_{1}+L_{2}+L_{3}\right)} \Sigma(k)=\sin ( & \left.k L_{1}\right) \sin \left(k L_{2}\right) \cos \left(k L_{3}\right) \\
& +\cos \left(k L_{1}\right) \sin \left(k L_{2}\right) \sin \left(k L_{3}\right)+\sin \left(k L_{1}\right) \cos \left(k L_{2}\right) \sin \left(k L_{3}\right) .
\end{aligned}
$$

5.4. Real secular determinant. Theorem 5.2 gives a handy tool for looking for the eigenvalues of a quantum graph. However, $\Sigma(k)$, as defined by equation (56) is a complex valued function (that needs to be evaluated on the real line - at least when looking for positive eigenvalues). A complex function is equal to zero when both real and imaginary part are equal to zero. It would be nicer to have one equation instead of two.

There are several indications that it should be possible. First, the fact that we do have roots of a complex function on the real line $\oint^{3}$ is atypical; it suggests that the function has some symmetries. Second, in the two examples that we considered in Section 5.3 we succeeded in making the secular determinant real. It turns out that the same method works in general.

\footnotetext{
${ }^{3}$ Since our operator is self-adjoint, the eigenvalues $\lambda=k^{2}$ must be real and therefore the roots of $\Sigma(k)$ are restricted to real and imaginary axes. Since the operator is bounded from below and unbounded from above, infinitely many of the eigenvalues must lie on the real axis
} 
Lemma 5.5. Let

$$
\mathcal{L}=\sum_{e \in E} L_{e}
$$

denote the total length of the graph. The analytic function

$$
\zeta(k)=\frac{e^{-i k \mathcal{L}}}{\sqrt{\operatorname{det} S}} \operatorname{det}(I-S D(k)),
$$

is real on the real line and has the same zeros as the secular determinant $\Sigma(k)$.

Proof. We remark that

$$
\operatorname{det}(D(k))=e^{2 i k \mathcal{L}}
$$

and the matrix $D(k)$ is unitary for real $k$. Denoting the unitary matrix $S D(k)$ by $U$, and using the identity $\operatorname{det}(A B)=\operatorname{det} A \operatorname{det} B$, we can rewrite

$$
\zeta(k)=(\operatorname{det} U)^{-1 / 2} \operatorname{det}(I-U),
$$

Using the unitarity of $U$, we now evaluate

$$
\begin{aligned}
\overline{\zeta(k)} & =(\operatorname{det} U)^{1 / 2} \operatorname{det}\left(I-U^{*}\right) \\
& =(\operatorname{det} U)^{1 / 2} \operatorname{det}(U-I) \operatorname{det} U^{*}=\zeta(k),
\end{aligned}
$$

where we used the identities

$$
I-U^{*}=U U^{*}-U^{*}=(U-I) U^{*} \text { and } \operatorname{det}\left(U^{*}\right)=(\operatorname{det} U)^{-1} .
$$

Exercise 5.6. Show that for a graph with Neumann conditions at every vertex except for $n$ vertices of degree 1 where Dirichlet conditions are imposed, the determinant of $S$ is $\operatorname{det} S=$ $(-1)^{|E|-|V|+n}$.

A detailed study of the secular determinant, including the interpretation of the coefficients of the polynomials like (58) and (60) in terms of special closed paths on the graph, appears in [6].

5.5. Remarks on numerical calculation of graph eigenvalues. Expression (63) which is guaranteed to be real for real $k$ allows for a simple way to compute eigenvalues of a quantum graph: find roots of a real function.

The naive method is to evaluate the function $\zeta(k)$ on a dense enough set of points to get bounds for the eigenvalues, within which the bisection method can be employed. The bisection method can be substituted by a more sophisticated tool such as Brent-Dekker method. For a small graph, the function $\zeta(k)$, which is a combination of trigonometric functions of incommensurate frequency, can be derived explicitly, giving access to its derivative and therefore Newton-like methods.

However, the initial evaluation may miss a pair of almost-degenerate eigenvalues. To check for this possibility, it is very effective to plot the difference between the counting function for the computed eigenvalues and the Weyl's Law. The approximate location of the missed eigenvalues (if any) can be seen very clearly, see Fig. 8 for a typical example. See also [48] for another method. 


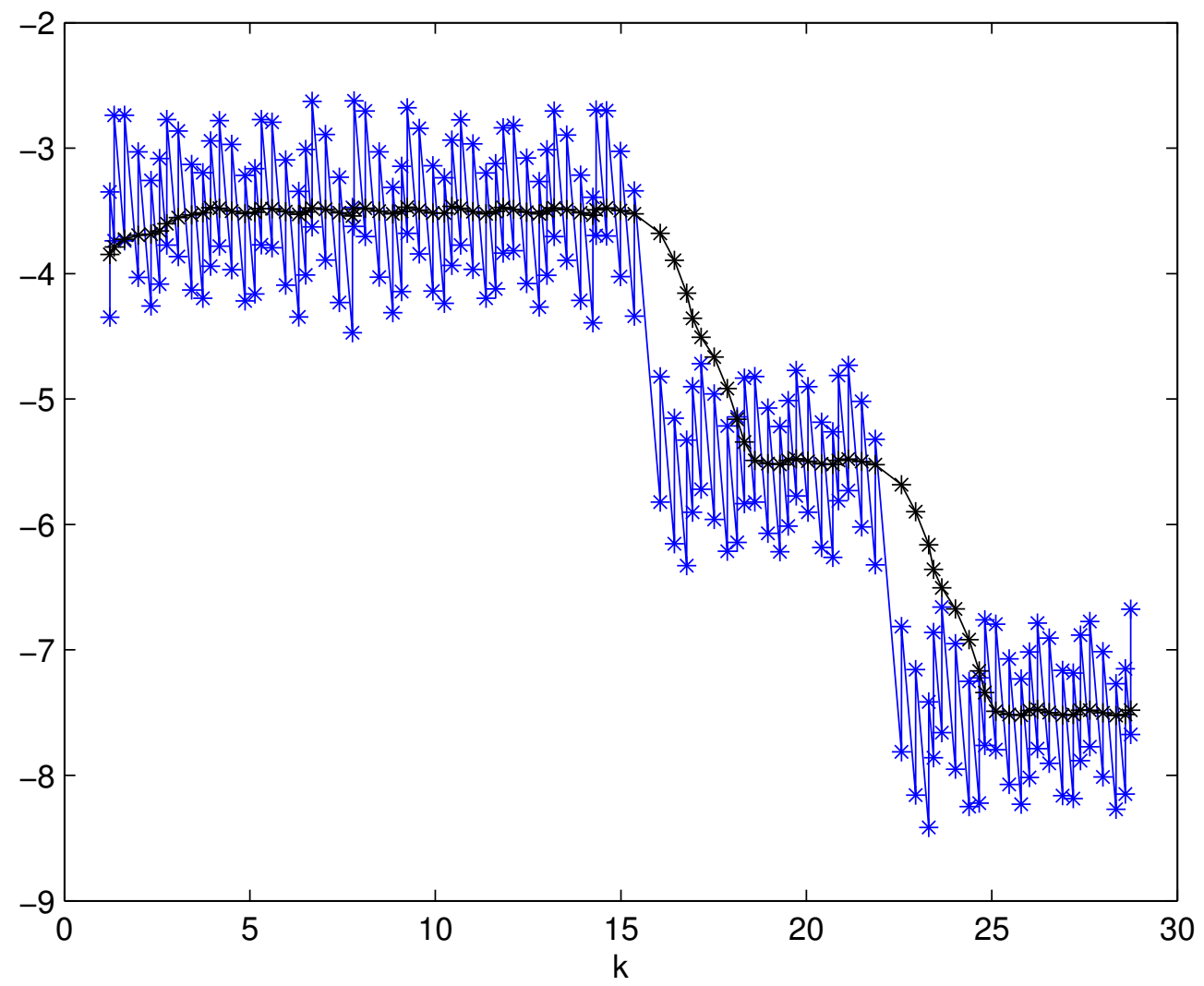

FiguRE 8. The difference between the counting function of the numerically found eigenvalue and the Weyl's estimate. The plot shows that two pairs of eigenvalues were missed, around $k=15.5$ and $k=22$.

A smarter method for the initial step is to use the interlacing inequalities of Lemma 4.1 to bracket the eigenvalues. Unfortunately, this involves computing eigenvalues of another graph, but it may be much simpler, as in the case of the star graphs.

\section{Symmetry AND isospeCtrality}

6.1. Example: 3-mandarin graph. The 3-mandarin graph is a graph with two vertices and three edges connecting them, see Fig. 9. If one is uncomfortable with multiple edges running between a pair of vertices, extra Neumann vertices of degree two may be placed on some edges, see Section 2.2 .

Labelling the edges running down by 1,2 and 3 , and using the ordering $[1,2,3, \overline{1}, \overline{2}, \overline{3}]$, the matrix $S$ becomes

$$
S=\left(\begin{array}{cccccc}
0 & 0 & 0 & -1 / 3 & 2 / 3 & 2 / 3 \\
0 & 0 & 0 & 2 / 3 & -1 / 3 & 2 / 3 \\
0 & 0 & 0 & 2 / 3 & 2 / 3 & -1 / 3 \\
-1 / 3 & 2 / 3 & 2 / 3 & 0 & 0 & 0 \\
2 / 3 & -1 / 3 & 2 / 3 & 0 & 0 & 0 \\
2 / 3 & 2 / 3 & -1 / 3 & 0 & 0 & 0
\end{array}\right)
$$




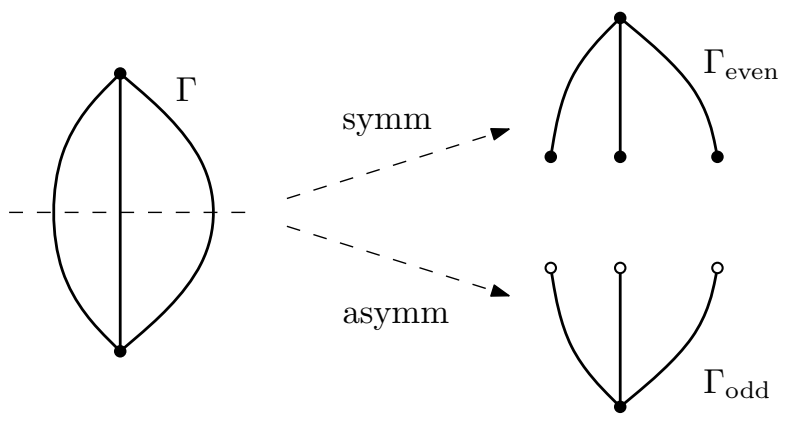

Figure 9. The mandarin graph with 3 edges (left) and its decomposition into the even and odd quotients (right). The Dirichlet vertices are distinguished as empty cicrles.

Denoting, as before, $z_{j}=e^{i k L_{j}}, j=1,2,3$, we get $D(k)=\operatorname{diag}\left(z_{1}, z_{2}, z_{3}, z_{1}, z_{2}, z_{3}\right)$. The secular determinant simplifies to

$$
\begin{aligned}
\Sigma(k)=\left(-z_{1} z_{2} z_{3}-\frac{1}{3}\left(z_{1} z_{2}+z_{2} z_{3}+z_{3} z_{1}\right)+\frac{1}{3}\left(z_{1}+z_{2}+z_{3}\right)+1\right) & \\
& \times\left(z_{1} z_{2} z_{3}-\frac{1}{3}\left(z_{1} z_{2}+z_{2} z_{3}+z_{3} z_{1}\right)-\frac{1}{3}\left(z_{1}+z_{2}+z_{3}\right)+1\right)
\end{aligned}
$$

where the factors coincide with the secular determinants we obtained for the star graphs with three edges and Neumann and Dirichlet conditions at the endpoints, correspondingly, see equations (58) and (60), modulo the change $z_{j}^{2} \leftrightarrow z_{j}$.

The reason for this factorization is the symmetry. The graph, as shown in Fig. 9, is symmetric with respect to the vertical (up-down) reflection. This means that the reflected eigenfunction is still an eigenfunction. Denote by $R f$ the reflected version of an eigenfunction $f$. By linearity, $f_{e}=f+R f$ and $f_{o}=f-R f$ satisfy the eigenvalue equation with the same $\lambda$. They may be identically zero, but not both at the same time, since $\left(f_{e}+f_{o}\right) / 2=f \not \equiv 0$. And under the action of $R, f_{e}$ is even and $f_{o}$ is odd:

$$
R f_{e}=f_{e}, \quad R f_{o}=-f_{o} .
$$

Using this idea one can show that every eigenspace has a basis of eigenfunctions each of which is either even or odd. Indeed, starting with an arbitrary basis of size $m$, we produce $2 m$ even/odd combinations from them. These combinations span the eigenspace (since $f=$ $\left.\left(f_{e}+f_{o}\right) / 2\right)$, so it remains to choose $m$ linearly independent vectors among them.

Consider an odd eigenfunction on the mandarin graph. Let $x_{m}$ be the midpoint of the first edge. This point is fixed under the action of reflection, thus $(R f)\left(x_{m}\right)=f\left(x_{m}\right)$. On the other hand, $R f=-f$, therefore $f\left(x_{m}\right)=-f\left(x_{m}\right)=0$. The same applies for the midpoint of every edge. Therefore every odd eigenfunction is the eigenfunction of the half of the mandarin with Dirichlet conditions at the midpoints. The converse is also true: starting from an eigenfunction of the half with Dirichlet boundary, we obtain an eigenfunction of the full graph by planting two copies and multiplying one of them by -1 .

A similar reasoning for even eigenfunctions of the mandarin graph shows that they are in one-to-one correspondence with the eigenfunctions of the half of the graph with Neumann 


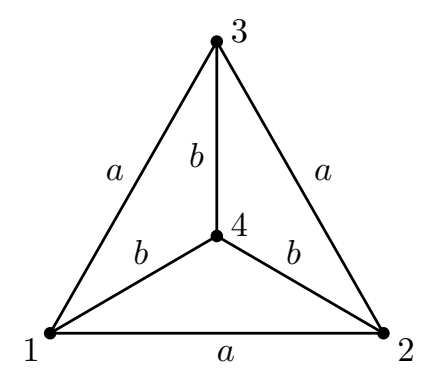

FiguRE 10. A tetrahedron graph which is invariant under rotation by $2 \pi / 3$ and horizontal reflection. Its group of symmetry is $S_{3}$ : an arbitrary permutation of vertices 1,2 and 3 .

boundary. The half of a 3-mandarin is a star graph with three edges, see Fig. 9; the edge lengths are half of the mandarin's.

It turns out that this symmetry of the mandarin graph (and the corresponding factorization (68) ) leads to interesting anomalies in the number of of zeros of the eigenfunctions. This subject will be visited again in Section 7.4 .

6.2. Quotient graphs. To put the observations of the previous section on a more formal footing, the Hilbert space $H(\Gamma)$ of functions on the mandarin graph (that are sufficiently smooth and satisfy correct vertex conditions) can be decomposed into the direct sum of two orthogonal subspaces $H_{o}$ and $H_{e}$, which are invariant with respect to operator $-d^{2} / d x^{2}$ acting on the graph. Restrictions of the operator to these subspaces can be identified with this operator acting on two smaller graphs, a star with Dirichlet ends and a star with Neumann ends.

Such a smaller graph, together with its vertex conditions, is called quotient graph and was introduced by Band, Parzanchevski and Ben-Shach [9, 42] to study isospectrality. To produce a quotient graph one needs a quantum graph, a group of symmetries (not necessarily the largest possible) and a representation of this group. We will not describe the full procedure, which can be learned from the already mentioned papers and the forthcoming article [3]. Instead we will briefly describe its consequences and explain one particular construction that leads to a pair of graphs with identical spectra (i.e. an isospectral pair).

Let $\Gamma$ be a graph with a finite group of symmetries $G$. To each irreducible representation $\rho$ there corresponds a subspace $H_{\rho}$ of the Hilbert space $H(\Gamma)$. This is the subspace of functions that transform according to the representation $\rho$ when acted upon by the symmetries from $G$. In some sources, such functions are called equivariant vectors; the subspace $H_{\rho}$ is called the isotypic component. The subspaces corresponding to different irreducible representations are orthogonal, the space $H(\Gamma)$ is a direct sum of $H_{\rho}$ over all irreps of the group $G$.

If $\rho$ has dimension $d>1$, then every eigenvalue of the Hamiltonian restricted to $H_{\rho}$ has multiplicity which is a multiple of $d$. Moreover, the secular determinant $\Sigma(k)$ factorizes into factors that correspond to the irreps $\rho$ of $G$. Each factor is raised to a power which is the dimension of the corresponding $\rho$ (hence the degeneracy of the corresponding eigenvalue),

$$
\Sigma_{\Gamma}(k)=\prod_{\text {irreps } \rho}\left(\Sigma_{\rho}(k)\right)^{\operatorname{dim}(\rho)} .
$$




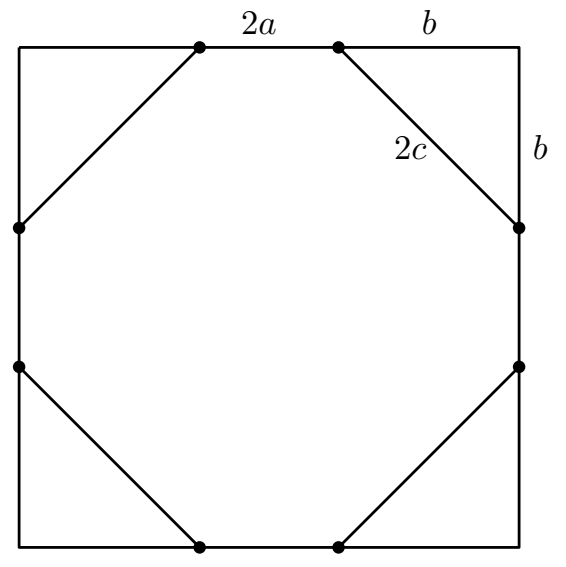

(a)

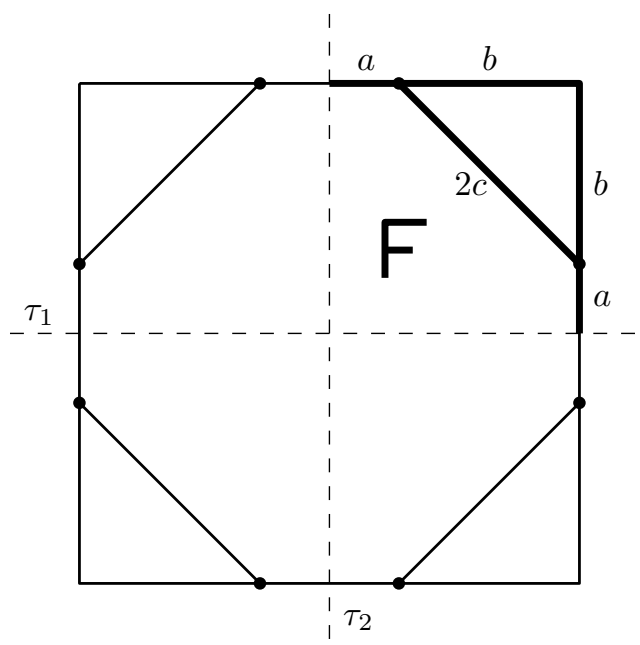

(b)

Figure 11. (a) A graph with symmetry group $D_{4}$, the dihedral group of degree 4; (b) symmetry axes of the first subgroup and a choice of fundamental domain.

Example 6.1 (from [3]). Consider the tetrahedron graph from Fig. 10. This graph is symmetric under reflection and rotation by $2 \pi / 3$. The corresponding group of symmetries is $S_{3}$, the group of permutations of 3 objects; in this case it can be thought of as permuting the vertices number 1, 2 and 3 . The group $S_{3}$ has three irreducible representations, trivial, alternating and $2 d$. The first two are one-dimensional, while the latter is 2-dimensional (as suggested by its name). The secular determinant $\Sigma(k)$ has the corresponding factorization

$$
\Sigma(k)=\frac{1}{27}\left(z_{a}-1\right)\left(3 z_{a} z_{b}^{2}-z_{b}^{2}+z_{a}-3\right)\left(3 z_{a}^{2} z_{b}^{2}+2 z_{a} z_{b}^{2}-z_{a}^{2}+z_{b}^{2}-2 z_{a}-3\right)^{2} .
$$

Note that it may happen that for some $\rho$ the subspace $H_{\rho}$ is trivial. In this case the corresponding factor $\Sigma_{\rho}(k)$ is 1 and there are no eigenvalues corresponding to this representation.

6.3. Example: dihedral graphs. The present example originates from [10] and is the origin of the theory of [9, 42]. Consider the graph on Fig. 11(a). It has the symmetries of the square, thus its full group of symmetries is the dihedral group of degree four $D_{4}$ (and order eight, hence another notation, $D_{8}$, which causes much confusion).

We will first consider the subgroup generated by the vertical (up-down) reflection $\tau_{1}$ and the horizontal (left-right) reflection $\tau_{2}$. One of the irreducible representations of this subgroup is

$$
\tau_{1} \mapsto(1), \quad \tau_{2} \mapsto(-1),
$$

where (1) (correspondingly $(-1)$ ) stands for the operation of multiplication by 1 (correspondingly -1$)$.

To understand the functions that transform according to representation (72), we choose as the fundamental domain (a subgraph that covers the entire graph under the action of the subgroup) the top right quarter of the graph shown in Fig. 11(b), and plant there a function $F$. Applying the vertical reflection $\tau_{1}$, we find that in the bottom right quarter of the graph, the function must be equal to $F$ multiplied by 1 and suitably reflected. Applying 


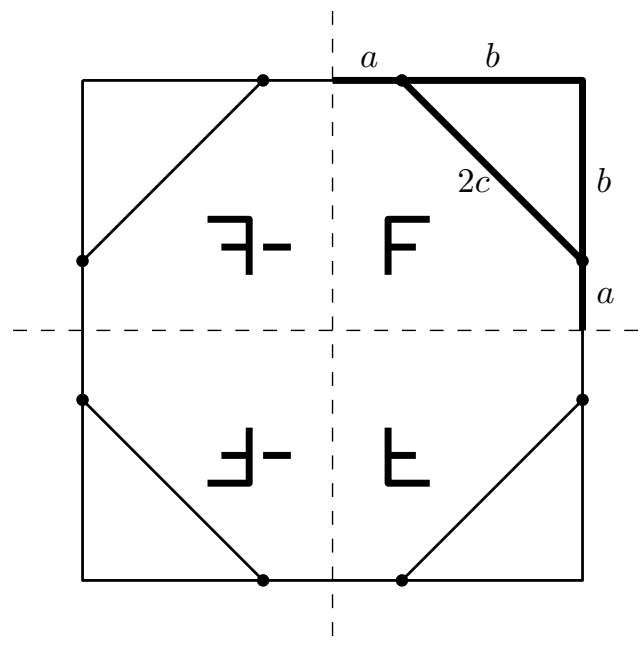

(a)

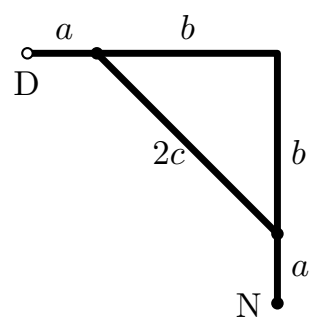

(b)

Figure 12. Constructing a quotient graph with respect to the representation (72).

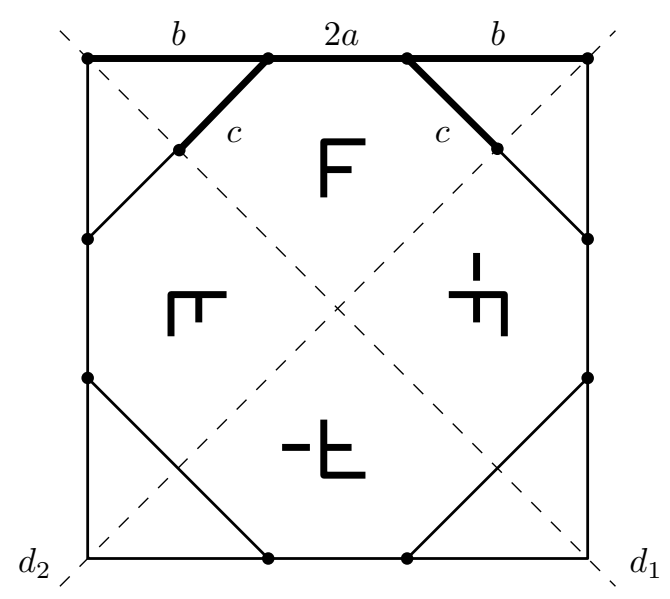

(a)

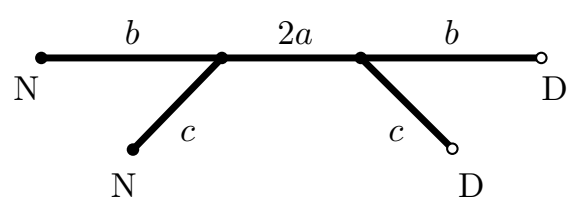

(b)

Figure 13. Constructing a quotient graph with respect to the representation (73).

the horizontal reflection $\tau_{2}$, we find that the left side of the graph must be populated by the copies of the function $F$ multiplied by -1 , see Fig. 12 (a).

We have already discovered in Section 6.1 that at the point where $F$ meets $-F$, the function must vanish (i.e. have the Dirichlet condition), whereas at the point where $F$ meets $F$ the condition must be Neumann. Thus we obtain the quotient graph in Fig. 12(c).

Let us now repeat the same procedure but choose the diagonal reflections $d_{1}$ and $d_{2}$ as the generators of the subgroup, together with the representation

$$
d_{1} \mapsto(1), \quad d_{2} \mapsto(-1) .
$$

Starting with a fundamental domain, reflecting and multiplying it as prescribed by (73), we fill the entire graph as in Fig. 13(a). The corresponding conditions on the fundamental domain are shown in Fig. $13(\mathrm{~b})$. 

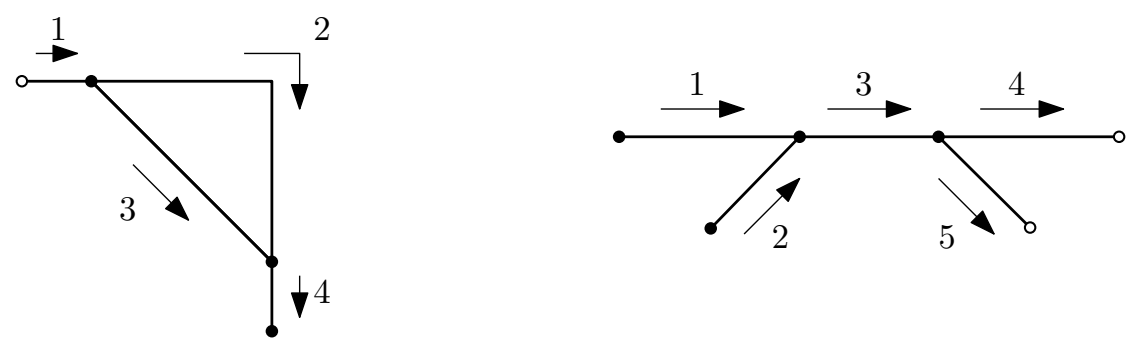

FIGURE 14. Numbering of edges of the two isospectral graph.

The most interesting feature of the two quotient subgraphs is that they are isospectral, i.e. have exactly the same eigenvalues. This can be shown by a transplantation procedure a la Buser [18, 19, which describes a unitary equivalence of the corresponding operators. Another possibility (admittedly more tedious) is to find the secular determinants of the two graphs.

To this end, number the edges of the two graphs as shown in Fig. 14. Starting with the graph with a cycle (which we will now call the dihedral graph, and its partner the dihedral tree), order its edges as $[1,2,3,4, \overline{1}, \overline{2}, \overline{3}, \overline{4}]$. Then

$$
S=\left(\begin{array}{cccccccc}
0 & 0 & 0 & 0 & -1 & 0 & 0 & 0 \\
2 / 3 & 0 & 0 & 0 & 0 & -1 / 3 & 2 / 3 & 0 \\
2 / 3 & 0 & 0 & 0 & 0 & 2 / 3 & -1 / 3 & 0 \\
0 & 2 / 3 & 2 / 3 & 0 & 0 & 0 & 0 & -1 / 3 \\
-1 / 3 & 0 & 0 & 0 & 0 & 2 / 3 & 2 / 3 & 0 \\
0 & -1 / 3 & 2 / 3 & 0 & 0 & 0 & 0 & 2 / 3 \\
0 & 2 / 3 & -1 / 3 & 0 & 0 & 0 & 0 & 2 / 3 \\
0 & 0 & 0 & 1 & 0 & 0 & 0 & 0
\end{array}\right)
$$

and $D(k)=\operatorname{diag}\left(z_{a}, z_{b}^{2}, z_{c}^{2}, z_{a}, z_{a}, z_{b}^{2}, z_{c}^{2}, z_{a}\right)$, where $z_{s}=e^{i k s}, s=a, b, c$. Evaluating the determinant, we obtain

$$
\Sigma(k)=-z_{a}^{4} z_{b}^{4} z_{c}^{4}+\frac{1}{9}\left(z_{a}^{4} z_{b}^{4}+z_{b}^{4} z_{c}^{4}+z_{c}^{4} z_{a}^{4}\right)+\frac{8}{9}\left(z_{a}^{4}-1\right) z_{b}^{2} z_{c}^{2}-\frac{1}{9}\left(z_{a}^{4}+z_{b}^{4}+z_{c}^{4}\right)+1 .
$$

Repeating the procedure for the dihedral tree, which has $10 \times 10$ matrices $S$ and $D(k)$, we arrive to the secular determinant which is again given by expression (75). It is easy to check that 0 is eigenvalue of neither graph and therefore the graphs are isospectral.

The glimpse of the underlying reason for the isospectrality can be seen in the secular determinant of the original graph (that of Fig. 11(a)). As predicted by (70), it factorizes:

$$
\begin{aligned}
\Sigma(k)= & \left(9 z_{a}^{4} z_{b}^{4} z_{c}^{4}-\left(z_{a}^{4} z_{b}^{4}+z_{b}^{4} z_{c}^{4}+z_{c}^{4} z_{a}^{4}\right)-8\left(z_{a}^{4}-1\right) z_{b}^{2} z_{c}^{2}+z_{a}^{4}+z_{b}^{4}+z_{c}^{4}-1\right)^{2} \\
& \times\left(3 z_{a}^{2} z_{b}^{2} z_{c}^{2}+z_{a}^{2} z_{b}^{2}+z_{b}^{2} z_{c}^{2}+z_{c}^{2} z_{a}^{2}-z_{a}^{2}-z_{b}^{2}-z_{c}^{2}-3\right) \\
& \times\left(3 z_{a}^{2} z_{b}^{2} z_{c}^{2}+z_{a}^{2} z_{b}^{2}-z_{b}^{2} z_{c}^{2}+z_{c}^{2} z_{a}^{2}-z_{a}^{2}+z_{b}^{2}+z_{c}^{2}+3\right) \\
& \times\left(3 z_{a}^{2} z_{b}^{2} z_{c}^{2}-z_{a}^{2} z_{b}^{2}+z_{b}^{2} z_{c}^{2}-z_{c}^{2} z_{a}^{2}-z_{a}^{2}+z_{b}^{2}+z_{c}^{2}-3\right) \\
& \times\left(3 z_{a}^{2} z_{b}^{2} z_{c}^{2}-z_{a}^{2} z_{b}^{2}-z_{b}^{2} z_{c}^{2}-z_{c}^{2} z_{a}^{2}-z_{a}^{2}-z_{b}^{2}-z_{c}^{2}+3\right)
\end{aligned}
$$

up to an overall factor. The last four terms correspond to the four one-dimensional representations of the group $D_{4}$, while the first one, squared, corresponds to the two-dimensional 
representation. The term inside the square also coincides with the secular determinant of the two quotient graphs. This suggests that although we constructed them as quotients by the (one-dimensional) representations of two different symmetry subgroups, they are both realizable as quotients by the two-dimensional representation of the whole group. This is indeed shown in [9], together with a general criterion for isospectrality involving induction of representations from subgroups to the whole group.

\section{Magnetic Schrödinger OPERATOR AND NODAL COUNT}

Magnetic field is introduced into the Schrödinger equation via the magnetic vector potential usually denoted $A(x)$. In our case, $A(x)$ is a one-dimensional vector: it changes sign if the direction of the edge is reversed. The Schrödinger eigenvalue equation then takes the form

$$
-\left(\frac{d}{d x}-i A(x)\right)^{2} f(x)+V(x) f(x)=k^{2} f(x),
$$

where the square is interpreted in the sense of operators, i.e.

$$
\begin{aligned}
\left(\frac{d}{d x}-i A(x)\right)^{2} f(x)=\left(\frac{d}{d x}-i A(x)\right) & \left(f^{\prime}(x)-i A(x) f(x)\right) \\
& =f^{\prime \prime}(x)-i(A(x) f(x))^{\prime}-i A(x) f^{\prime}(x)-A^{2}(x) f(x) .
\end{aligned}
$$

To understand the "strange" definition of $A(x)$ a little better, consider the equation

$$
-\left(\frac{d}{d x}-i A\right)^{2} f(x)=k^{2} f(x)
$$

where $A$ is a "normal" constant, on the interval $[0, L]$. Solutions of this equation, $e^{ \pm i k x+i A x}$, under the change of variables $x \mapsto L-x$ become solutions of a slightly different equation,

$$
-\left(\frac{d}{d x}+i A\right)^{2} f(L-x)=k^{2} f(L-x) .
$$

But this variable change is just a reparametrization of the interval in terms of the distance from the other end and should not affect the laws of physics. Letting $A$ to be the "one-form" which transforms according to $A(L-x)=-A(x)$ addresses this problem.

To understand the effect of the magnetic potential $A(x)$ on the secular determinant derived in Section 5.2, we write the solution of (76) with $V \equiv 0$ on the $j$-th edge in the form

$$
f_{j}(x)=a_{j} e^{i k x+i \int_{0}^{x} A(x)}+a_{j} e^{i k\left(L_{j}-x\right)+i \int_{L_{j}}^{x} A(x)} .
$$

Taking $A(x)$ to be the constant vector on the edge (as we will see, this results in no loss of generality), we obtain a somewhat more manageable form

$$
f_{j}(x)=a_{j} e^{i(k+A) x}+a_{j} e^{i(k-A)\left(L_{j}-x\right)},
$$

which also highlights the fact that the wave travelling in the negative direction (the second term) feels the magnetic potential as $-A$.

The vertex conditions also change with the introduction of the magnetic field. It is convenient to define the operator

$$
D=\frac{d}{d x}-i A(x)
$$


so that the Schrödinger operator can be written as $-D^{2}+V$ and the Neumann vertex conditions become

$$
\begin{aligned}
& f(x) \text { is continuous, } \\
& \sum_{e \sim v} D f(v)=0,
\end{aligned}
$$

which is to be compared to (2)-(3).

Applying these conditions to the solution form (81), we get

$$
\begin{array}{r}
a_{1}+a_{\bar{j}} e^{i k L_{1}+i A_{\overline{1}} L_{1}}=\ldots=a_{d}+a_{\bar{d}} e^{i k L_{d}+i A_{\bar{d}} L_{d}}, \\
\sum_{j=1}^{d} a_{j}-\sum_{j=1}^{d} a_{\bar{j}} e^{i k L_{j}+i A_{\bar{j}} L_{j}}=0,
\end{array}
$$

which assumes that all edges attached to $v$ are oriented outward and also introduces the notation $A_{\bar{j}}=-A_{j}$ in agreement with the nature of $A$. In fact the same answer would be obtained had we started with equation (80) instead, and defined

$$
A_{j}=\frac{1}{L_{j}} \int_{0}^{L_{j}} A(x), \quad A_{\bar{j}}=\frac{1}{L_{j}} \int_{L_{j}}^{0} A(x) .
$$

Either way, the only change in the definition of the secular determinant $\Sigma(k)=\operatorname{det}(I-S D(k))$ is in the diagonal matrix $D(k)$ which becomes

$$
D(k)_{b, b}=e^{i k L_{b}+i \int_{b} A(x)} .
$$

As we just saw, the precise nature of $A(x)$ is not important; the only quantity that enters $\Sigma(k)$ is the integral of $A(x)$. In fact, even more is true: only the values of the integral of $A(x)$ around the cycles of the graph are important.

Definition 7.1. The flux of the magnetic field given by $A(x)$ through an oriented cycle $\gamma$ of $\Gamma$ is the integral of $A(x)$ over the cycle,

$$
\alpha_{\gamma}=\int_{\gamma} A(x)
$$

Theorem 7.2. Consider two operators on the same quantum graph $\Gamma$ differing only in their magnetic potentials $A_{1}(x)$ and $A_{2}(x)$. Then they are unitarily equivalent if their fluxes through every cycle on $\Gamma$ are equal modulo $2 \pi$. In fact, it is enough to compute the fluxes through a fundamental set of $\beta=|E|-|V|+1$ cycles. A magnetic perturbation of a Schrödinger operator on a graph $\Gamma$ is thus fully specified by a set of $\beta$ numbers between $-\pi$ and $\pi$.

The proof of this simple theorem can be found in, for example, [34] or [15], Section 2.6.

7.1. Example: Dihedral graph. To study the influence of the magnetic perturbation on the eigenvalues of the dihedral graph, we put the magnetic flux $\alpha$ through its loop by using $D(k)=\operatorname{diag}\left(z_{a}, e^{i \alpha} z_{b}^{2}, z_{c}^{2}, z_{a}, z_{a}, e^{-i \alpha} z_{b}^{2}, z_{c}^{2}, z_{a}\right)$ together with the matrix $S$ as given in (74). This results in the secular determinant

$$
\Sigma(k)=-z_{a}^{4} z_{b}^{4} z_{c}^{4}+\frac{1}{9}\left(z_{a}^{4} z_{b}^{4}+z_{b}^{4} z_{c}^{4}+z_{c}^{4} z_{a}^{4}\right)+\frac{8}{9} \cos (\alpha)\left(z_{a}^{4}-1\right) z_{b}^{2} z_{c}^{2}-\frac{1}{9}\left(z_{a}^{4}+z_{b}^{4}+z_{c}^{4}\right)+1 .
$$




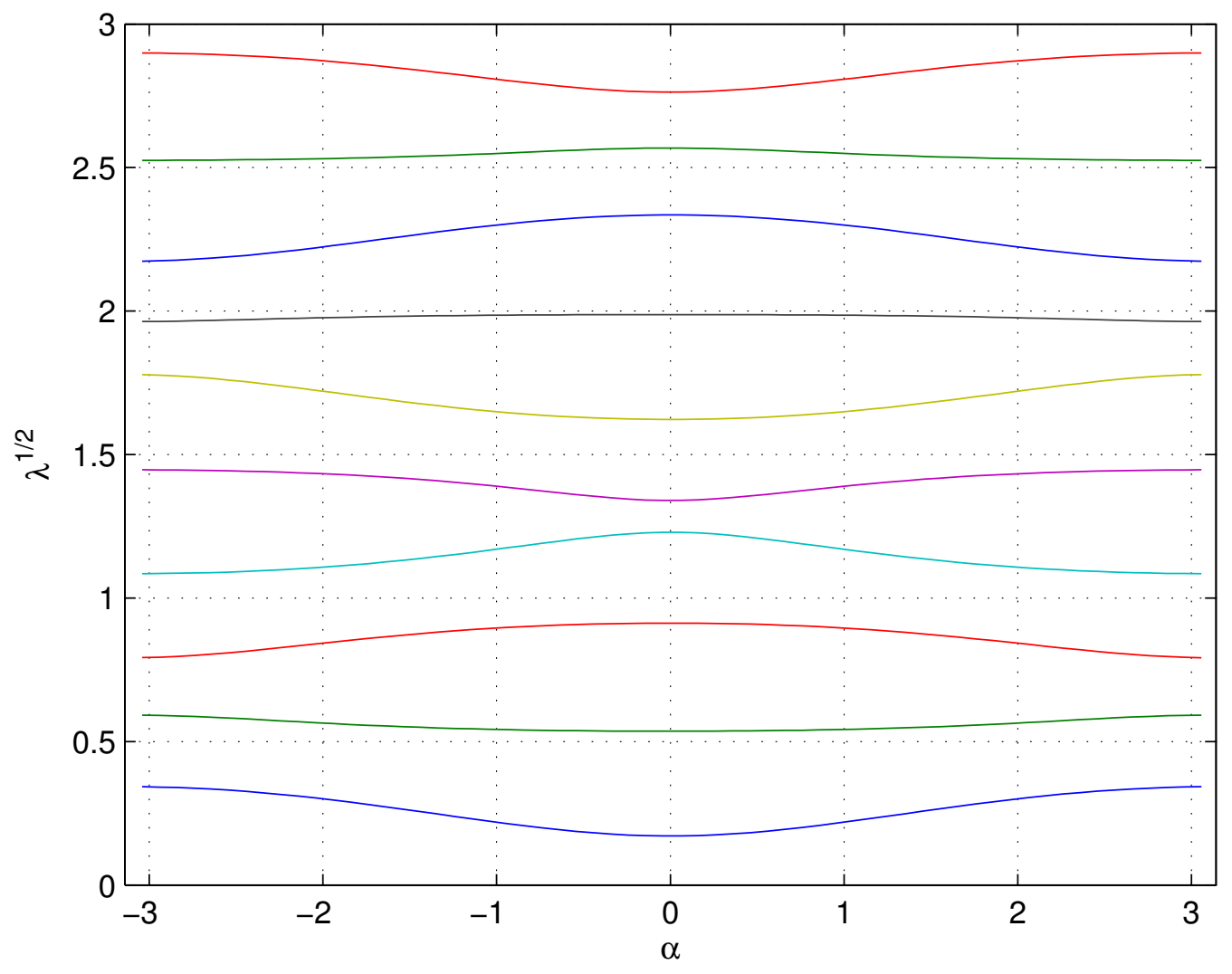

FiguRE 15. The square roots of the first few eigenvalues of the dihedral graph as function of the magnetic flux through the cycle. The lengths are $a=\pi$, $b=1, c=\sqrt{2}$.

We plot the first few eigenvalues $\lambda_{n}(\alpha)$ of the resulting graph as a function of the magnetic flux $\alpha$, Fig. [15. Since the flux is only important modulo $2 \pi$, we plot the eigenvalues over the interval $[-\pi, \pi]$. We observe that the eigenvalues are symmetric (even) functions with respect to $\alpha=0$ : this can be seen directly by complex conjugating equation (76): if $f(x)$ is an eigenfunction with potential $A(x)$ then $\overline{f(x)}$ is an eigenfunction with potential $-A(x)$ with the same eigenvalue. Sometimes the eigenvalue has a minimum at $\alpha=0$ and sometimes a maximum. These events do not alternate as in the Hill's equation 4 there is no strict periodicity there, but we will be able to extract some information about them.

7.2. Magnetic-nodal connection. Let us for a moment come back to the case of no magnetic field, $\alpha=0$, and study the eigenfunctions of the dihedral graph, see Fig. 16. In Table 1 we give the results of a numerical calculation for one choice of the graph's lengths. We list the sequential number of the eigenvalue, starting from 1, its value, the number of zeros of the corresponding eigenfunction, and, somewhat arbitrarily, a description of the behavior of the

\footnotetext{
${ }^{4}$ Hill's differential equation 39 is a Schrödinger equation on $\mathbb{R}^{1}$ with periodic potential; its Floquet reduction is equivalent to a quantum graph in the shape of a circle with a magnetic flux $\alpha$ through it. It is an important result of Hill's equation theory that the minima and maxima of $\lambda_{n}(\alpha)$ at $\alpha=0$ alternate with $n$.
} 

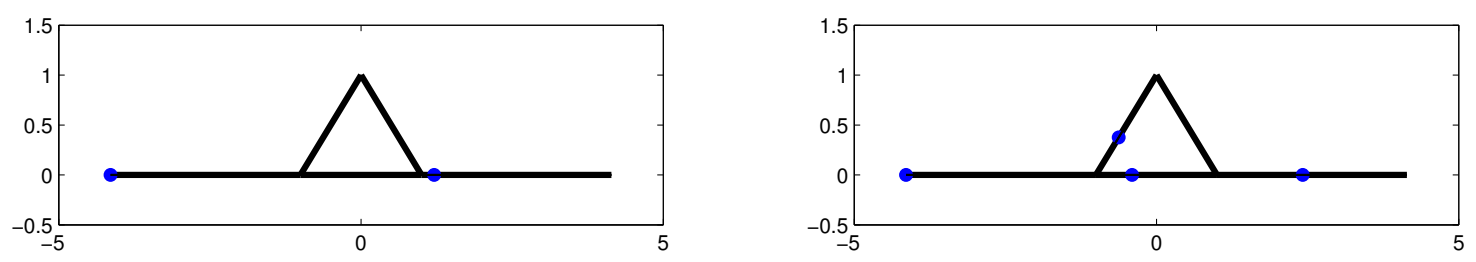

FiguRE 16. Location of zeros of two eigenfunctions of the dihedral graph. Eigenfunctions number $n=2$ and $n=3$ are displayed. The zero at the left endpoint is due to the Dirichlet condition; such zeros are not counted when we report the nodal count of an eigenfunction.

eigenvalue of the magnetic dihedral graph (see Fig. 15), namely whether it has a maximum or a minimum at $\alpha=0$.

\begin{tabular}{l|ccccccccc}
$n$ & 1 & 2 & 3 & 4 & 5 & 6 & 7 & 8 & 9 \\
\hline$\lambda_{n}(0)$ & 0.1708 & 0.5359 & 0.9126 & 1.2294 & 1.3398 & 1.6225 & 1.9877 & 2.3349 & 2.5680 \\
$\#$ zeros & 0 & 1 & 3 & 4 & 4 & 5 & 7 & 8 & 9 \\
$\lambda_{n}(\alpha)$ at 0 & min & $\min$ & $\max$ & $\max$ & $\min$ & $\min$ & $\max$ & $\max$ & $\max$
\end{tabular}

TABLE 1. Eigenvalues of a dihedral graph with the lengths $a=\pi, b=1$ and $c=\sqrt{2}$, the number of zeros of the corresponding eigenfunction, and the behavior of the magnetic eigenvalue around the point of no magnetic field.

A careful reader will observe a curious pattern: the magnetic eigenvalue $\lambda_{n}(\alpha)$ appears to have a minimum whenever the number of zeros of the eigenfunction, which we will denote $\phi_{n}$ is less than $n$ and a maximum whenever $\phi_{n}=n$. The connection between the two was discovered in [13] for discrete Laplacians, an alternative proof given in [20] and an extension to quantum graphs proved in [17].

Before we formulate this result we need to recall some definitions from multivariate calculus.

Definition 7.3. Let $F\left(x_{1}, \ldots, x_{\beta}\right)$ be a twice differentiable function of $\beta$ variables.

- The point $x^{*}=\left(x_{1}^{*}, \ldots, x_{\beta}^{*}\right)$ is a critical point of the function $F$ if all first derivatives of $F$ vanish at $x^{*}$,

$$
\frac{\partial F}{\partial x_{j}}\left(x_{1}^{*}, \ldots, x_{\beta}^{*}\right)=0 .
$$

- The Hessian matrix of $F$ at $x^{*}$ is the matrix of all second derivatives of $F$,

$$
\operatorname{Hess}(F)=\left(\frac{\partial^{2} F}{\partial x_{j} \partial x_{k}}\right)_{j, k=1}^{\beta} .
$$

The matrix $\operatorname{Hess}(F)$ is symmetric therefore all of its eigenvalues are real.

- A critical point is called non-degenerate if the Hessian evaluated at the point is a non-degenerate matrix (has no zero eigenvalues).

- The Morse index of a critical point is the number of the negative eigenvalues of its Hessian. The Second Derivative Test says that if the Morse index of a non-degenerate critical point is zero, the point is a local minimum; if it equals the dimension of the space, the point is a maximum. 
Theorem 7.4 (Berkolaiko-Weyand [17]). Let $\Gamma^{\alpha}$ be a quantum graph with magnetic Schrödinger operator characterized by magnetic fluxes $\boldsymbol{\alpha}=\left(\alpha_{1}, \ldots, \alpha_{\beta}\right)$ through a fundamental set of cycles. Let $\psi$ be the eigenfunction of $\Gamma^{0}$ that corresponds to a simple eigenvalue $\lambda=\lambda_{n}\left(\Gamma^{0}\right)$ of the graph with zero magnetic field. We assume that $\psi$ is non-zero on vertices of the graph.

Then $\boldsymbol{\alpha}=(0, \ldots, 0)$ is a non-degenerate critical point of the function $\lambda_{n}(\boldsymbol{\alpha}):=\lambda_{n}\left(\Gamma^{\boldsymbol{\alpha}}\right)$ and its Morse index is equal to the nodal surplus $\phi-(n-1)$, where $\phi$ is the number of internal zeros of $\psi$ on $\Gamma$.

As a corollary we get a simple but useful bound on the number of zeros of $n$-th eigenfunction.

Corollary 7.5. Let $\Gamma$ be a quantum graph with a Schrödinger operator, $\lambda_{n}$ be its $n$-th eigenvalue and $\psi_{n}$ the corresponding eigenfunction. If $\lambda_{n}$ is simple and $\psi_{n}$ does not vanish on vertices, the number of zeros $\phi_{n}$ of the function $\psi_{n}$ is a well-defined quantity which satisfies

$$
0 \leq \phi_{n}-(n-1) \leq \beta=|E|-|V|+1 \text {. }
$$

Remark 7.6. The result of Corollary 7.5 actually predates Theorem 7.4 by a considerable time. It goes back to the results on trees [44, 49], their extension to $\beta>0$ for nodal domains [12] and to number of zeros [4]. But it also follows easily from Theorem 7.4 due to the Morse index being an integer between 0 and the dimension of the space of parameters.

Exercise 7.7. Let $\Gamma$ be a quantum graph with a Schrödinger operator, $\lambda$ be its simple eigenvalue and $\psi$ the corresponding eigenfunction which does not vanish on vertices. Prove that $\psi$ has an even number of zeros on every cycle of the graph $\Gamma$.

7.3. Nodal count of the dihedral graph. It turns out there is an explicit formula for the number of zeros of the $n$-th eigenfunction of the dihedral graph. This formula was discovered by Aronovitch, Band, Oren and Smilansky [8]. The discovery was remarkable as no explicit formula for the eigenvalues of the dihedral graph is known.

The formula was proved in [4] using a fairly involved construction of opening the graph by attaching two phase-synchronized infinite leads. It would be nice to be able to prove this result from Theorem 7.4 directly, but we know of no such proof. Instead, we will give here a relatively simple proof showcasing the power of the interlacing results of Section 4.1 , Corollary 7.5 and the simple observation that the number of zeros on any cycle of the graph must be even. This proof has not previously appeared in any other source.

Theorem 7.8 (Conjectured in [8], first proved in [4]). Let $n$-th eigenvalue of a dihedral graph be simple and the corresponding eigenfunction not vanish at the vertices (except at the Dirichlet vertex). Then the number of zeros of the eigenfunction is

$$
\phi_{n}=n-\bmod _{2}\left(\left\lfloor\frac{b+c}{a+b+c} n\right\rfloor\right) .
$$

Remark 7.9. It can be shown that the hypothesis of the theorem is satisfied for all eigenvalues and eigenfunction for a generic choice of lengths $a, b$ and $c$ [16].

It is interesting to observe that the sequence of nodal counts $\left\{\phi_{n}\right\}$ contains the information about the relative length of the central loop in the graph.

Before we can prove the theorem, we need two auxiliary lemmas. 


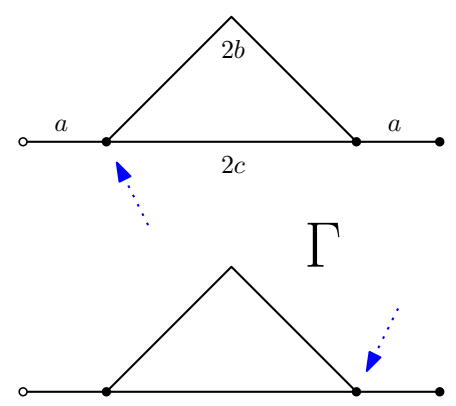

(a)

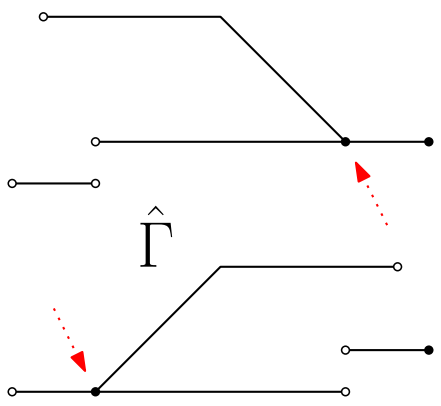

(b)

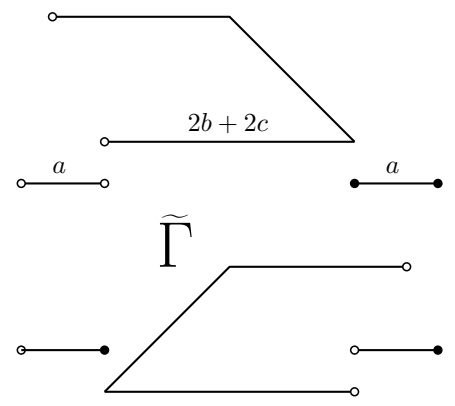

(c)

Figure 17. Modifications of the graph $\Gamma$, consisting of two copies of the dihedral graph, leading to interlacing inequalities (95) and (96). The Dirichlet vertices are distinguished as empty cicrles.

Lemma 7.10. Let $\lambda_{n}$ denote the eigenvalue of the dihedral graph with parameters $a, b$ and $c$ and let $\tilde{\lambda}_{n}$ denote the ordered numbers from the set

$$
\tilde{\sigma}:=\left\{\frac{\pi}{2 a} n_{1}\right\}_{n_{1} \in \mathbb{N}} \cup\left\{\frac{\pi}{2(b+c)} n_{2}\right\}_{n_{2} \in \mathbb{N}} .
$$

Then

$$
\lambda_{n} \leq \tilde{\lambda}_{n} \leq \lambda_{n+1}, \quad n=1,2, \ldots
$$

Proof. Denote by $\sigma$ the spectrum of the dihedral graph. Consider the following sequence of modifications of the graph $\Gamma$ consisting of two copies of the dihedral graph, Fig. 17(a). First we impose the Dirichlet condition on the left attachment point of one copy and the right attachment point of the other copy, obtaining the graph $\hat{\Gamma}$, see Fig. 17 (b). Second we separate the right edge of the first dihedral graph and the left edge of the second dihedral graph, imposing Neumann conditions on the newly formed vertices, see Fig. 17(c). The first modification is covered by two applications of Lemma 4.1, while the second by two applications of Lemma 4.3 (in reverse).

Observe that the spectrum of the graph $\Gamma$ is $\sigma \cup \sigma$ (in the sense of multisets) and the spectrum of the final graph $\widetilde{\Gamma}$ is $\{0\} \cup \tilde{\sigma} \cup \tilde{\sigma}$. We will denote the eigenvalues of the three stages by $s_{n}, \hat{s}_{n}$ and $\tilde{s}_{n}$ correspondingly. Because of the degeneracies in the spectrum and the interlacing Lemmas, we have the following inequalities,

$$
0<s_{2}=s_{1} \leq \hat{s}_{1} \leq \hat{s}_{2} \leq s_{4}=s_{3} \leq \hat{s}_{3} \leq \hat{s}_{4} \leq \ldots
$$

and

$$
\tilde{s}_{1}=0<\hat{s}_{1} \leq \tilde{s}_{3}=\tilde{s}_{2} \leq \hat{s}_{2} \leq \hat{s}_{3} \leq \tilde{s}_{5}=\tilde{s}_{4} \leq \hat{s}_{4} \leq \ldots
$$

Combining the two, we obtain

$$
\tilde{s}_{1}=0<s_{2}=s_{1} \leq \tilde{s}_{3}=\tilde{s}_{2} \leq s_{4}=s_{3} \leq \tilde{s}_{5}=\tilde{s}_{4} \leq \ldots
$$

Now the claim of the Lemma follows by observing that $s_{1}=\lambda_{2}, \tilde{s}_{2}=\tilde{\lambda}_{1}, s_{4}=\lambda_{2}, \tilde{s}_{4}=\tilde{\lambda}_{2}$ etc. 
Lemma 7.11. Let $S_{1}=\left\{\frac{n_{1}}{\alpha}\right\}_{n_{1} \in \mathbb{N}}$ and $S_{2}=\left\{\frac{n_{2}}{\beta}\right\}_{n_{2} \in \mathbb{N}}$ with positive $\alpha$ and $\beta$. If

$$
\#\left\{S_{1} \cup S_{2} \leq \lambda\right\}=n-1 \quad \text { then } \quad \#\left\{S_{1} \leq \lambda\right\}=\left\lfloor\frac{\alpha}{\alpha+\beta} n\right\rfloor .
$$

Remark 7.12. This Lemma was offered as an exercise to the attendees of 2015 Séminaire de Mathématiques Supérieures "Geometric and Computational Spectral Theory" at CRM, University of Montreal. At that time, the author did not know a good proof.

Several students, including Luc Petiard, Arseny Rayko, Lise Turner and Saskia Voß submitted proofs. The proof below is based on Arseny's proof with elements borrowed from other attendees' versions.

Proof. Let

$$
\#\left\{S_{1} \leq \lambda\right\}=\lfloor\lambda \alpha\rfloor=: N_{1}, \quad \#\left\{S_{2} \leq \lambda\right\}=\lfloor\lambda \beta\rfloor=: N_{2},
$$

then $N_{1}+N_{2}=n-1$ and also

$$
\begin{aligned}
& N_{1} \leq \lambda \alpha<N_{1}+1 \\
& N_{2} \leq \lambda \beta<N_{2}+1
\end{aligned}
$$

Multiplying the first inequality by $\beta$ and the second by $\alpha$ and going through the middle term, we get

$$
\beta N_{1}<\alpha\left(N_{2}+1\right), \quad \alpha N_{2}<\beta\left(N_{1}+1\right) .
$$

Adding $\alpha N_{1}$ to the first and $\alpha\left(N_{1}+1\right)$ to the second inequalities we get

$$
(\alpha+\beta) N_{1}<\alpha\left(N_{1}+N_{2}+1\right)<(\alpha+\beta)\left(N_{1}+1\right) .
$$

Dividing by $\alpha+\beta$ and using $N_{1}+N_{2}+1=n$ we get

$$
N_{1}<\frac{\alpha}{\alpha+\beta} n<N_{1}+1
$$

which implies the desired result.

Proof of Theorem 7.8. The eigenvalue $\lambda_{n}$ of the dihedral graph has $n-1$ eigenvalues from $\tilde{\sigma}$ below it, so by Lemma 7.11, applied with $\alpha=2 a / \pi$ and $\beta=2(b+c) / \pi$ it has $N_{1}=\left\lfloor\frac{a}{a+b+c} n\right\rfloor$ eigenvalues from the sequence $\left\{\frac{\pi}{2 a}\right\}$, which are precisely the eigenvalues of the side edges of the dihedral graph but with the Dirichlet conditions at the attachment points (i.e. the eigenvalues of the signle edge components of the graph $\hat{\Gamma}$ ).

By Sturm's theorem, the eigenfunction of the dihedral graph corresponding to $\lambda_{n}$ has $N_{1}$ zeros on the side edges. Therefore $\phi_{n}-N_{1}$ zeros lie on the cycle and this quantity must be even. But we know from Corollary 7.5 that $\phi_{n}$ is either $n-1$ or $n$. To make $\phi_{n}-N_{1}$ even we must choose

$$
\phi_{n}=n-\bmod _{2}\left(n-N_{1}\right)=n-\bmod _{2}\left(\left\lfloor\frac{b+c}{a+b+c} n\right\rfloor\right) .
$$




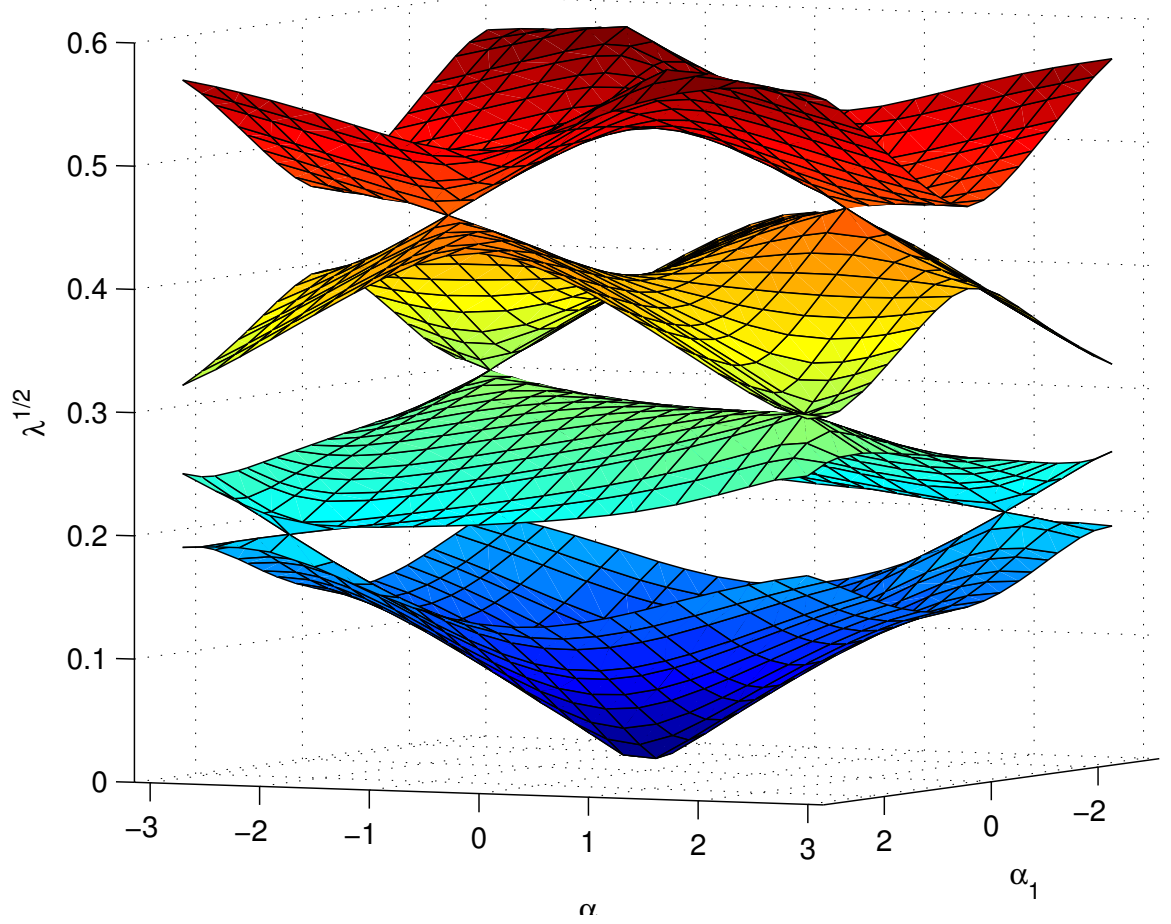

FiguRE 18. The first four eigenvalues of the mandarin graph as functions of two magnetic fluxes $\alpha_{1}$ and $\alpha_{2}$. The surfaces can be seen to be touching at conical singularities, the so-called Dirac points.

7.4. Nodal count of a mandarin graph. When the bounds of equation (91) were discovered, the following question arose: for an arbitrary graph, do all allowed numbers in the range $0,1, \ldots, \beta$ appear as the nodal surplus $\phi_{n}-(n-1)$ of some eigenfunction? It turns out the answer is no.

Theorem 7.13 (Band-Berkolaiko-Weyand [5]). Let $\Gamma$ be a mandarin graph of 2 vertices connected by $d$ edges. If the eigenvalue number $n>1$, is simple and the corresponding eigenfunction does not vanish on vertices, then the nodal surplus $\sigma_{n}$ satisfies

$$
1 \leq \sigma_{n} \leq \beta-1 \text {. }
$$

In particular, the nodal surplus of the 3-mandarin graph is equal to 1 for all eigenfunctions except the first, whose nodal surplus is always 0.

It is interesting to combine this observation with the result of Theorem 7.4. The Morse index is never equal to 0 (for $n>1$ ) or to the dimension $\beta$, therefore the point $\boldsymbol{\alpha}=0$ is never a minimum or a maximum. But the space of all possible magnetic fluxes is a $\beta$-dimensional torus, which is compact. Therefore the extrema must be achieved somewhere!

There are other "standard" points where the eigenvalue $\lambda_{n}(\boldsymbol{\alpha})$ always have a critical point. It is relatively straightforward to extend Theorem 7.4 to points $\left(b_{1}, \ldots, b_{\beta}\right)$, where each $b_{i}$ is 0 or $\pi$. But it is also straightforward to extend Theorem 7.13 (see [5] for both extensions) which will show that those critical point are also never extrema.

The missing extrema turn out to be achieved at singular points of $\lambda_{n}(\boldsymbol{\alpha})$, see Fig. 18 for an example. Such conical singularities are sometimes called the Dirac points and their 
appearance in 3-mandarin graphs is intimately related to their appearance in the dispersion relation of graphene [38]. This connection, however, lies outside the scope of this article.

\section{Concluding Remarks}

There are many interesting topics within the area of spectral theory of metric graphs that we did not cover. A very partial list (heavily biased towards personal preferences of the author) is as follows:

- Generic properties of eigenfunctions and eigenvalues. Many results, such as Theorem 7.4 require the eigenvalue to be simple and the eigenfunction to be non-vanishing on vertices. These properties are generic with respect to small perturbations of the edge lengths (that need to break all symmetries of the graph). Results in this direction can be found in [27], 21] (for eigenvalues) and [16] (for both eigenvalues and eigenfunctions).

- Ergodic flow on the secular manifold. Barra and Gaspard [11] introduced an interpretation of the secular determinant equation as an ergodic flow piercing a compact manifold (more precisely, an algebraic variety) given by solutions of an equation like (75) on a torus. This interpretation leads to many surprising and very general results, including those of [2] and [21].

- Spectral theory of infinite periodic graphs yields a fruitful connection to the theory of compact graphs with magnetic field. The background is covered in Chapter 4 of [15]; a sample of results can be found in [31, 23], [38], and [2, 5].

- Graphs make a very interesting setting to study resonances. Work in this direction has attracted many researchers from across the field [37, [50, [22, [35], [28, [24, 25, 26].

- There is an ongoing effort to find bounds on the graph eigenvalues (especially the lowest non-zero ones) in terms of the geometric properties of the graph, see [33, 17, 1] for the latest results and references.

\section{REFERENCES}

[1] S. Ariturk. Eigenvalue estimates on quantum graphs. preprint arXiv:1609.07471, 2016.

[2] R. Band and G. Berkolaiko. Universality of the momentum band density of periodic networks. Phys. Rev. Lett., 111:130404, Sep 2013.

[3] R. Band, G. Berkolaiko, C. Joyner, and W. Liu. Symmetry of quantum graphs and factorization of the spectral determinant. in preparation, 2016.

[4] R. Band, G. Berkolaiko, and U. Smilansky. Dynamics of nodal points and the nodal count on a family of quantum graphs. Annales Henri Poincare, 13(1):145-184, 2012.

[5] R. Band, G. Berkolaiko, and T. Weyand. Anomalous nodal count and singularities in the dispersion relation of honeycomb graphs. J. of Math. Phys., 56(12):122111, 2015.

[6] R. Band, J. M. Harrison, and C. H. Joyner. Finite pseudo orbit expansions for spectral quantities of quantum graphs. J. Phys. A, 45(32):325204, 19, 2012.

[7] R. Band and G. Lévy. Quantum graphs which optimize the spectral gap. preprint arXiv:1608.00520, 2016.

[8] R. Band, I. Oren, and U. Smilansky. Nodal domains on graphs - how to count them and why? In Analysis on graphs and its applications, volume 77 of Proc. Sympos. Pure Math., pages 5-27. Amer. Math. Soc., Providence, RI, 2008.

[9] R. Band, O. Parzanchevski, and G. Ben-Shach. The isospectral fruits of representation theory: quantum graphs and drums. J. Phys. A, 42(17):175202, 42, 2009.

[10] R. Band, T. Shapira, and U. Smilansky. Nodal domains on isospectral quantum graphs: the resolution of isospectrality? J. Phys. A, 39(45):13999-14014, 2006. 
[11] F. Barra and P. Gaspard. On the level spacing distribution in quantum graphs. J. Statist. Phys., 101(12):283-319, 2000.

[12] G. Berkolaiko. A lower bound for nodal count on discrete and metric graphs. Comm. Math. Phys., 278(3):803-819, 2008.

[13] G. Berkolaiko. Nodal count of graph eigenfunctions via magnetic perturbation. Anal. PDE, 6:1213-1233, 2013. preprint arXiv:1110.5373.

[14] G. Berkolaiko and P. Kuchment. Dependence of the spectrum of a quantum graph on vertex conditions and edge lengths. In Spectral Geometry, volume 84 of Proceedings of Symposia in Pure Mathematics. American Math. Soc., 2012. preprint arXiv:1008.0369.

[15] G. Berkolaiko and P. Kuchment. Introduction to Quantum Graphs, volume 186 of Mathematical Surveys and Monographs. AMS, 2013.

[16] G. Berkolaiko and W. Liu. Simplicity of eigenvalues and non-vanishing of eigenfunctions of a quantum graph. J. Math. Anal. Appl., 445(1):803-818, 2017. preprint arXiv:1601.06225.

[17] G. Berkolaiko and T. Weyand. Stability of eigenvalues of quantum graphs with respect to magnetic perturbation and the nodal count of the eigenfunctions. Philos. Trans. R. Soc. Lond. Ser. A Math. Phys. Eng. Sci., 372(2007):20120522, 17, 2014.

[18] P. Buser. Isospectral Riemann surfaces. Ann. Inst. Fourier (Grenoble), 36(2):167-192, 1986.

[19] P. Buser, J. Conway, P. Doyle, and K.-D. Semmler. Some planar isospectral domains. Internat. Math. Res. Notices, (9):391-400, 1994.

[20] Y. Colin de Verdière. Magnetic interpretation of the nodal defect on graphs. Anal. PDE, 6:1235-1242, 2013. preprint arXiv: 1201.1110.

[21] Y. Colin de Verdière. Semi-classical measures on quantum graphs and the Gauß map of the determinant manifold. Annales Henri Poincaré, 16(2):347-364, 2015. also arXiv:1311.5449.

[22] E.B. Davies and A. Pushnitski. Non-Weyl resonance asymptotics for quantum graphs. Analysis \& PDE, 4:729-756, 2011.

[23] P. Exner, P. Kuchment, and B. Winn. On the location of spectral edges in $\mathbb{Z}$-periodic media. J. Phys. A, 43(47):474022, 8, 2010.

[24] P. Exner and J. Lipovský. Equivalence of resolvent and scattering resonances on quantum graphs. In Adventures in mathematical physics, volume 447 of Contemp. Math., pages 73-81. Amer. Math. Soc., Providence, RI, 2007.

[25] P. Exner and J. Lipovský. Resonances from perturbations of quantum graphs with rationally related edges. J. Phys. A, 43(10):105301, 21, 2010.

[26] P. Exner and J. Lipovský. Non-Weyl resonance asymptotics for quantum graphs in a magnetic field. Phys. Lett. A, 375(4):805-807, 2011.

[27] L. Friedlander. Genericity of simple eigenvalues for a metric graph. Israel J. Math., 146:149-156, 2005.

[28] S. Gnutzmann, H. Schanz, and U. Smilansky. Topological resonances in scattering on networks (graphs). Phys. Rev. Lett., 110:094101, Feb 2013.

[29] S. Gnutzmann and U. Smilansky. Quantum graphs: Applications to quantum chaos and universal spectral statistics. Adv. Phys., 55(5-6):527-625, 2006.

[30] Daniel Grieser. Monotone unitary families. Proc. Amer. Math. Soc., 141(3):997-1005, 2013. preprint arXiv:0711.2869.

[31] J. M. Harrison, P. Kuchment, A. Sobolev, and B. Winn. On occurrence of spectral edges for periodic operators inside the Brillouin zone. J. Phys. A, 40(27):7597-7618, 2007.

[32] R. A. Horn and C. R. Johnson. Matrix analysis. Cambridge University Press, Cambridge, second edition, 2013.

[33] J. B. Kennedy, P. Kurasov, G. Malenová, and D. Mugnolo. On the spectral gap of a quantum graph. Ann. Henri Poincaré, 17(9):2439-2473, 2016.

[34] V. Kostrykin and R. Schrader. Quantum wires with magnetic fluxes. Comm. Math. Phys., 237(1-2):161179, 2003. Dedicated to Rudolf Haag.

[35] T. Kottos and H. Schanz. Statistical properties of resonance widths for open quantum graphs. Waves Random Media, 14(1):S91-S105, 2004. Special section on quantum graphs.

[36] T. Kottos and U. Smilansky. Quantum chaos on graphs. Phys. Rev. Lett., 79(24):4794-4797, 1997.

[37] T. Kottos and U. Smilansky. Chaotic scattering on graphs. Phys. Rev. Lett., 85(5):968-971, 2000. 
[38] P. Kuchment and O. Post. On the spectra of carbon nano-structures. Comm. Math. Phys., 275(3):805826, 2007.

[39] Wilhelm Magnus and Stanley Winkler. Hill's equation. Interscience Tracts in Pure and Applied Mathematics, No. 20. Interscience Publishers John Wiley \& Sons New York-London-Sydney, 1966.

[40] D. Mugnolo. Semigroup methods for evolution equations on networks. Understanding Complex Systems. Springer, Cham, 2014.

[41] S. Nicaise. Some results on spectral theory over networks, applied to nerve impulse transmission. In Orthogonal polynomials and applications (Bar-le-Duc, 1984), volume 1171 of Lecture Notes in Math., pages 532-541. Springer, Berlin, 1985.

[42] O. Parzanchevski and R. Band. Linear representations and isospectrality with boundary conditions. $J$. Geom. Anal., 20(2):439-471, 2010.

[43] L. Pauling. The diamagnetic anisotropy of aromatic molecules. J. Chem. Phys., 4(10):673-677, 1936.

[44] Yu. V. Pokornyı̆, V. L. Pryadiev, and A. Al'-Obeĭd. On the oscillation of the spectrum of a boundary value problem on a graph. Mat. Zametki, 60(3):468-470, 1996.

[45] O. Post. Spectral Analysis on Graph-like Spaces, volume 2039 of Lecture Notes in Mathematics. Springer Verlag, Berlin, 2012.

[46] J.-P. Roth. Spectre du laplacien sur un graphe. C. R. Acad. Sci. Paris Sér. I Math., 296(19):793-795, 1983.

[47] K. Ruedenberg and C. W. Scherr. Free-electron network model for conjugated systems. i. J. Chem. Phys., 21(9):1565-1581, 1953.

[48] H. Schanz. A relation between the bond scattering matrix and the spectral counting function for quantum graphs. In Quantum graphs and their applications, volume 415 of Contemp. Math., pages 269-282. Amer. Math. Soc., Providence, RI, 2006.

[49] P. Schapotschnikow. Eigenvalue and nodal properties on quantum graph trees. Waves Random Complex Media, 16(3):167-178, 2006.

[50] C. Texier and G. Montambaux. Scattering theory on graphs. J. Phys. A, 34(47):10307-10326, 2001.

[51] J. von Below. A characteristic equation associated to an eigenvalue problem on $c^{2}$-networks. Linear Algebra Appl., 71:309-325, 1985.

Department of Mathematics, Texas A\&M University, College Station, TX 77843-3368, USA 